Review Article

\title{
Chinese Herbal Medicine for Postpartum Depression: A Systematic Review of Randomized Controlled Trials
}

\author{
Yongle Li, ${ }^{1,2}$ Zijie Chen, ${ }^{1}$ Ning Yu, ${ }^{1}$ Keyu Yao, ${ }^{1}$ Yiwen Che, \\ Yupeng $\mathrm{Xi}^{1,3}$ and Shuangqing $\mathrm{Zhai}^{1}$ \\ ${ }^{1}$ School of Preclinical Medicine, Beijing University of Chinese Medicine, Beijing 100029, China \\ ${ }^{2}$ College of Traditional Chinese Medicine, Inner Mongolia Medical University, Hohhot 010110, China \\ ${ }^{3}$ Eye Hospital, China Academy of Chinese Medical Sciences, Beijing 100040, China \\ Correspondence should be addressed to Shuangqing Zhai; zsq2098@163.com
}

Received 14 February 2016; Revised 26 July 2016; Accepted 10 August 2016

Academic Editor: Mark Moss

Copyright (C) 2016 Yongle Li et al. This is an open access article distributed under the Creative Commons Attribution License, which permits unrestricted use, distribution, and reproduction in any medium, provided the original work is properly cited.

Background. Postpartum depression (PPD) does great harm to women following childbirth. The aim of this study was to conduct a systematic review of the literature to assess the efficacy and safety of CHM for the treatment of PPD. Methods. Published or ongoing registered trials were searched for from the inception of the various databases to December 31, 2015. Data extraction and methodology assessment were conducted independently by two researchers. RevMan 5.3 software was used to analyze the data. Results. Forty-seven registered clinical trials (RCTs) were identified and reviewed. The results showed CHM alone or in combination with routine treatments could reduce HAMD score, EPDS score, incidence of adverse events, TESS, and SERS. CHM combined with routine treatment was more effective in increasing serum estradiol levels and reducing progesterone levels than routine treatment alone. Meanwhile, pooled data revealed that MRLQS combined with routine treatments or MRLQS plus MSHS combined with routine treatments were more effective than other therapeutic methods in TCM. MRLQS plus MSHS alone was found to be an effective alternative when compared to routine treatments. Conclusions. This review suggested that CHM was safe and effective in the treatment of PPD. However, this could not be proven conclusively. To ensure evidence-based clinical practice, more rigorously designed trials are warranted.

\section{Background}

Childbirth is an important experience for women, during which great physical and psychological changes can occur. Postpartum depression (PPD) is a common complication of childbirth, mainly affecting a woman's mentality during the first postpartum year [1]. It is accompanied by changes in thought and behavior and physical symptoms as well. It may negatively affect a woman's quality of life, limit her capacity to fulfill her maternal role, impair mother-infant bonding, and strain relationship with her partner. It even has negative influences on the child's emotional, cognitive, social, and behavioral developments [2-5]. The prevalence of PPD was estimated to be much greater than $10 \%-15 \%$ [1]. Some studies have reported that $60 \%$ or more of mothers of newborns were affected in certain areas $[6,7]$.
The etiology of PPD is multifactorial. Psychological and sociological changes are important risk factors. A personal and family history of depression, poor marital relationship, poor social support, stressful life events, low socioeconomic status, unplanned/unwanted pregnancy, and regional culture may increase the risk for this disease [8-10]. In addition, the reported trigger for the development of PPD refers to biological changes, such as estrogen and progesterone balance, hypothalamic-pituitary-adrenal axis, serotonergic neurotransmitter system, and certain genetic variations [1115].

Due to differing causative factors, the treatments for PPD are diverse. Selective serotonin reuptake inhibitors (SSRIs) and serotonin-norepinephrine reuptake inhibitors (SNRIs) are antidepressants used as first-line agents. Hormone treatments are considered to be an alternative method 
of pharmacotherapy. Psychotherapeutic and psychosocial interventions are the important nonpharmacotherapeutic methods used in clinics. Although various therapeutic approaches are available, sufficient evidence is not available to justify the use of any one of these approaches for PPD in clinical practice $[1,16-19]$.

Chinese herbal medicine (CHM), which is the most important component of traditional Chinese medicine (TCM), is widely used in China and is increasingly being used worldwide. According to TCM theory, the etiology of PPD mainly includes stagnation of the liver, stasis of the blood, deficiency of liver blood, phlegm-dampness, deficiency of kidney yin, deficiency of heart yin and blood, and deficiency in the heart and spleen [20-26]. The clinical treatments of TCM should conduct syndrome differentiation (bian zheng) and project corresponding therapeutic methods to prescription Chinese medicine formula according to analysis of etiology and syndrome. Many clinical trials involving CHM for the treatment of PPD have been conducted and report promising results. Although a meta-analysis (involving 9 trials and 716 patients) based on integrated traditional Chinese and Western medicine for the treatment of PPD has been reported in a Chinese journal [27], its applicability is limited due to its focus on integrative medicine, and only the overall response rate was analyzed in the literature. A comprehensive summary of the safety and efficacy of CHM for the treatment of PPD does not currently exist. Therefore, we systematically reviewed the literature to assess this aspect and to justify the use of CHM for the treatment of PPD in clinical practice.

\section{Methods}

The protocol used for this systematic review was published in the PROSPERO database (http://www.crd.york.ac.uk/ PROSPERO/display_record.asp?ID=CRD42014013258).

2.1. Eligibility Criteria. Randomized controlled trials (RCTs) testing the effectiveness of CHM in the treatment of PPD in women were included. There was no limitation on language, blinding, or publication format. Quasi-randomized trials were excluded. A clear description of the diagnostic criteria used must have been included. The patients included in this review were at least 16 years of age. CHM included the use of a single herb, Chinese patent medicine, practitioner-prescribed herbal formula, or products extracted from natural herbs. There was no limitation on dosage, administration regimen, or duration of treatment. The control could be a placebo, no treatment, or routine treatments. Trials evaluating the use of $\mathrm{CHM}$ combined with routine treatments versus the routine treatments alone were also included. The primary outcome measured was improvement in depression at the end of treatment or at the time of follow-up. Valid assessment tools included the Hamilton Depression Rating Scale (HAMD), Edinburgh Postnatal Depression Score (EPDS), Self-Rating Depression Scale (SDS), Beck Depression Inventory (BDI), Postpartum Depression Screening Scale (PDSS), and adverse events associated with CHM. Additionally, some studies measured changes in the levels of estradiol and progesterone.
2.2. Search Strategy and Study Selection. Two authors (Zijie Chen and Ning $\mathrm{Yu}$ ) conducted a systematic search for published, unpublished, and ongoing trials. The Cochrane Central Register of Controlled Trials (CENTRAL), PubMed, Excerpta Medica database (EMBASE), Chinese Biomedical Literature Database (CBM), Chinese National Knowledge Infrastructure Database (CNKI), Chinese Science and Technology Periodical Database (VIP), and Wan Fang Database were searched from the date of their inception until December 31, 2015. The World Health Organization (WHO) International Clinical Trial Registry Platform (ICTRP) portal and the website of International Clinical Trial Registry by US National Institutes of Health (http://clinicaltrials.gov/) were searched for ongoing registered clinical trials.

The following search terms were used individually or combined: "maternal depression", "post partum depression", "postpartum depression", "puerperium depression", "postnatal depression", "Chinese traditional", "Chinese herb", "Oriental traditional", "herb", "herbal medicine", and "random". There were no restrictions on language, publication year, and publication status. The details were shown in Supplementary Table 1 in Supplementary Material available online at http://dx.doi.org/10.1155/2016/5284234.

Titles and abstracts were screened to identify trials that potentially met the criteria of this study. The full texts of selected trials were reviewed by two authors (Zijie Chen and Ning $\mathrm{Yu}$ ) to determine eligibility for inclusion. The reference lists for all eligible articles were perused to collect additional trials. Any discrepancies were resolved by consensus and discussion with a third party (Shuangqing Zhai) if necessary.

2.3. Data Extraction. Data information was extracted independently by two authors (Keyu Yao and Yupeng Xi). A predesigned structured data extraction form was used for the eligible trials, which mainly included publication year, study type, random sequence generation, allocation concealment, blinding, diagnostic criteria, sample size, baseline characteristics of the participants, type of syndrome differentiation, therapeutic methods of TCM, intervention, control, outcomes, follow-up, and adverse events. The authors of the trials were contacted if any information was unclear or missing. Disagreements were resolved by discussion and consensus was reached with the help of a third party (Shuangqing Zhai).

2.4. Assessment of Risk of Bias. The risk of bias in eligible trials was assessed independently by two authors (Keyu Yao and Yupeng $\mathrm{Xi}$ ) according to the criteria described in the Cochrane Handbook version 5.1.0 [73]. The criteria mainly included random method, allocation concealment, blinding of participants and personnel, blinding of outcome assessment, incomplete outcome data, selective reporting, and other sources of bias. Each trial was classified as having a low, unclear, or high risk of bias. Any discrepancies were settled by discussion with a third party (Shuangqing Zhai). If necessary, the author of the trial was contacted to clarify any ambiguities. 
2.5. Data Analysis. RevMan 5.3 software was used to analyze the data. If the data were available, the following comparisons were performed: CHM versus no treatments, $\mathrm{CHM}$ versus placebo, and CHM with or without routine treatments versus routine treatments alone. The diversity of therapeutic methods used in TCM may be an important reason for the high heterogeneity in the interventions, and the subgroups were further analyzed in this review. A meta-analysis was performed if homogeneity in study design, participants, intervention, control, and outcome measures was acceptable. A random-effect model was used for the meta-analysis if the trials had significant heterogeneity $\left(I^{2}>50 \%\right)$ [74]. Otherwise, a fixed-effect model was used. The effect estimate was expressed as risk ratio (RR) with a 95\% confidence interval (CI) for categorical data and as mean difference (MD) with a 95\% CI for continuous data. Funnel plots were generated to detect publication bias when more than ten trials were identified [74].

\section{Results}

3.1. Description of Studies. The initial search yielded 17,640 records from seven electronic databases: CNKI $(n=2,237)$, CBM $(n=4,302)$, VIP $(n=5,245)$, Wan Fang $(n=5,853)$, PubMed $(n=3)$, CENTRAL $(n=0)$, and EMBASE $(n=0)$. After removing 9,132 records, which were duplicates from the different databases, 8,508 citations were screened. 8,289 records were excluded for various reasons determined while reading the titles and abstracts. Full text of the remaining 219 articles was retrieved and assessed in detail for eligibility. An additional 172 trials were excluded due to inappropriate interventions, diagnostic uncertainty, basic/theory researches, review, duplicate publication, absence of randomized controlled trials, or nonavailability of outcomes. Ultimately, 47 RCT articles [21, 26, 28-72] met the inclusion criteria and were included in this systematic review. A flow chart detailing the search process and study selection is displayed in Figure 1. We also searched trial registries that identified only one ongoing clinical trial (http://clinicaltrials.gov/). However the trial (ClinicalTrials.gov Identifier: NCT01178008) focused on acupuncture for the treatment of PPD and did not meet our criteria for inclusion.

All 47 trials were conducted and published in China; 44 were journal articles (93.62\%) [21, 26, 28-46, 48-53, 55, 56, $58-72]$ and 3 were postgraduate papers $(6.38 \%)$ [47, 54, 57]. All of the trials included two arms with the exception of one [63]. That trial included four arms. The main characteristics of the included trials are displayed in Table 1. A total of 3,795 participants (1,959 in intervention groups and 1,836 in control groups) with PPD and ranging in age from 18 to 43 years old $[41,48]$ were included. The sample sizes ranged from 45 to $150[42,62]$. Baseline demographics were provided in all of the studies except one [69], and all reported no significant differences between the intervention and control groups. To obtain the diagnostic criteria for PPD, 36 trials used DSM-IV [21, 26, 30-32, 34-38, 40, 42-45, 47, 48, 5059, 61-63, 66-68, 70-72], 9 trials used CCMD-III [28, 29, $33,39,46,49,60,65,69]$, one trial used ICD-X [64], and one trial used both of CCMD-III and DSM-V [41]. Nine trials provided information on the patients' syndrome differentiation (bianzheng fenxing) $[28,46-48,57,58,60,63$, $68]$, and 18 trials $[26,52,54-57,61,63,67,68,72]$ provided TCM therapeutic methods used to formulate individualized herbal preparations.

There were 2 trials $[57,63]$ that evaluated CHM versus a placebo, 9 trials $[34,35,42,50,62,64,65,71,72]$ that evaluated CHM versus routine treatments, and 36 trials [21, 26, 28$33,36-41,43-49,51-56,58-61,66-70]$ that evaluated CHM plus routine treatments versus routine treatments alone. 39 different types of CHM were investigated. The specific compositions of CHM are shown in Table 2. All types of CHM could be categorized into eight categories according to the therapeutic methods of TCM, as is shown in Table 2. The routine treatments mainly consisted of SSRIs (e.g., fluoxetine hydrochloride, paroxetine hydrochloride), SNRIs (e.g., citalopram, duloxetine, venlafaxine, sertraline), TCAs (e.g., amitriptyline), and psychotherapeutic and psychosocial interventions. The total treatments duration varied from 4 weeks to 6 months $[47,62]$.

All trials included primary outcomes and secondary outcomes. 34 trials [21, 28, 31, 35-42, 44-50, 52, 54, 55, 57, $58,60,61,64-72]$ reported depression scores measured by HAMD or EPDS. Four trials $[47,57,59,63]$ reported changes in estradiol and progesterone concentrations. 39 trials [21, 26, 29, 30, 32-39, 41-44, 46, 49-57, 59-69, 71, 72] reported adverse events. Among these, 30 trials [26, 29-32, 34-36, $41-43,46,49-55,57,59,60,62-66,69,71,72]$ reported incidence of adverse events, 6 trials [33, 37, 39, 61, 64, 69] used Treatment Emergent Symptoms Scale (TESS), and 2 trials $[38,44]$ used Rating Scale for Side Effects (SERS) to assess the safety of CHM for the treatment of PPD. Only 3 trials $[32,43,49]$ conducted follow-up examinations after the end of treatment.

3.2. Methodological Quality. All the trials provided limited information about design and methodology. The randomized allocation of participants among the groups was mentioned in all trials, but only 20 trials $[21,26,30,33,35,38,41,45,49$, $50,52,54,57,60,63,65,67,68,70,71]$ described the specific methods used for sequence generation. These included random number table, drawing of lots, and randomized card law. Allocation concealment was only reported in one trial [57]; however it could not be judged whether this was properly conducted because of insufficient information. Only one trial [63] was of double-blind design, and the rest of trials did not provide any information regarding blinding. Information detailing withdrawal from the study was provided in 13 trials $[29,37,39,43,45,47,52,54,57,63,67,68,72]$; of these, 3 trials $[45,47,67]$ reported that no participants dropped out, 3 trials [52, 57, 68] provided withdrawal reasons, and only one trial [57] conducted intention-to-treat analysis. To assess selective reporting, we searched the included registration information using trial registries. Registration information and the protocol used for the study were not accessible for all trials. Therefore, we assessed selective reporting by comparing the outcome measures mentioned in materials 


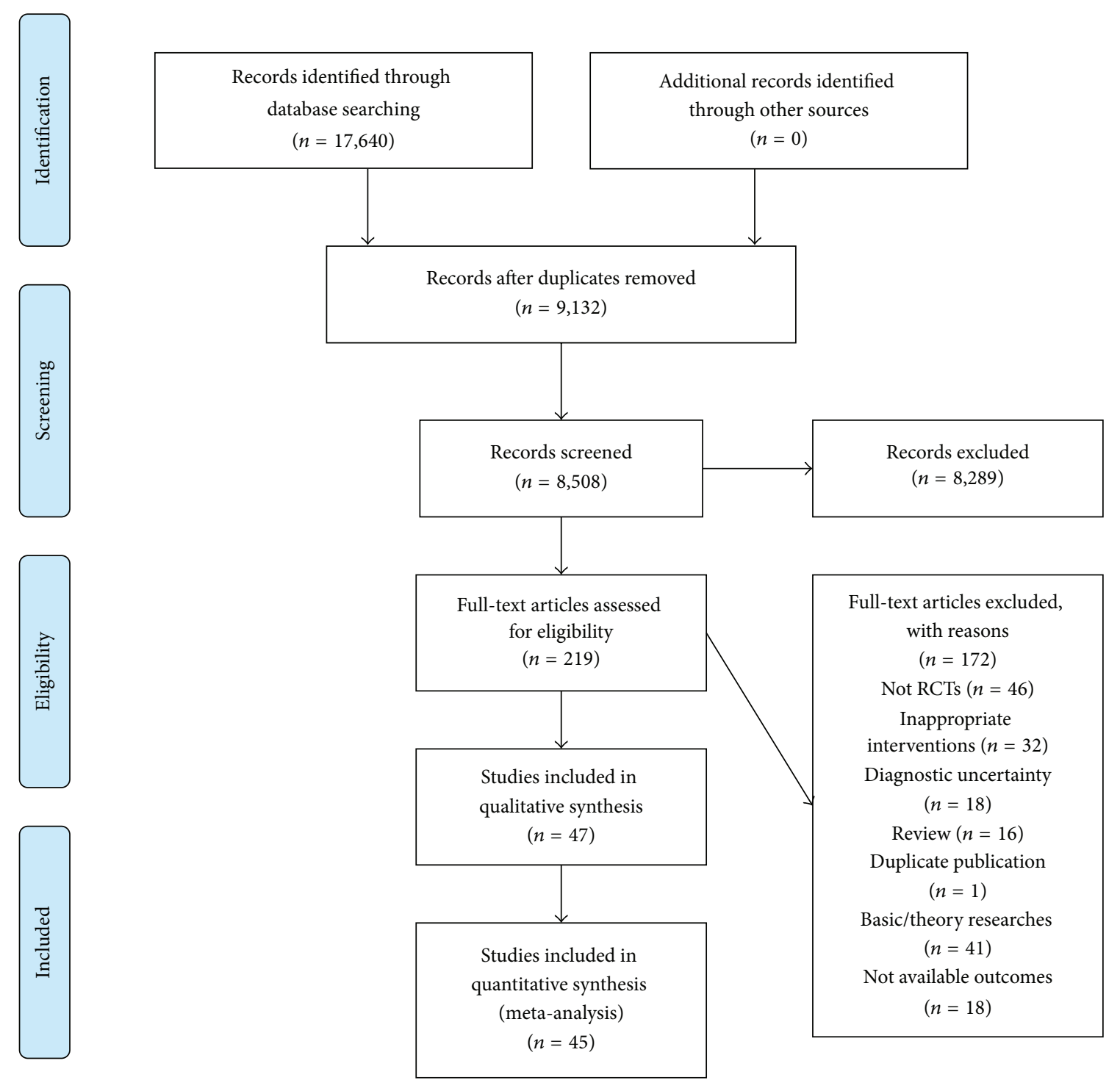

FIGURE 1: Flow diagram of the search process and study selection for the systematic review.

and methods section with the outcomes reported in the results. 20 trials $[26,28,33,36,38,41,42,44,47,48,50,54,57$, $58,60,62,64,67,68,72]$ were evaluated as low risk because all of the reported outcomes were described in the methods, 4 trials $[45,46,63,66]$ were evaluated as high risk because the outcomes reported in the results did not match prespecified outcomes, and 23 trials [29-32, 34, 35, 37, 39, 40, 43, 49, 51$56,59,61,65,67,69-71]$ were evaluated as unclear because no description of outcomes was provided in the materials and methods section. Only one trial [68] conducted a pretrial estimation of required sample size. Consequently, all of the trials had a high risk of bias and were assessed to be of low quality (Figure 2 and Supplementary Figure 1).

3.3. Effect Estimates. Among the 47 included trials, the possibility of pooling the effect estimates was limited due to differences in the methods of intervention used. Therefore, the findings were reported under three categories including CHM versus placebo (2 trials), CHM versus routine treatments (9 trials), and CHM plus routine treatments versus routine treatments alone (36 trials). Additionally, TCM therapeutic methods were further studied by subgroup analysis. Although few trials described the therapeutic methods of TCM used, they could be deduced by CHM component. The deductions were performed by two authors (Yongle $\mathrm{Li}$ and Yiwen Che), and any disagreements were resolved with the help of a third party (Shuangqing Zhai). The therapeutic methods were divided into the method of relieving liver qi stagnation (MRLQS) (shu gan jie yu fa), the method of strengthening heart and spleen (MSHS) (bu yi xin pi fa), the method of blood-activating and stasis-dissolving (MBASD) (huo xue hua yu fa), the method of tonifying qi and blood (MTQB) (bu yi qi xue fa), or a combination of the methods to illustrate the existence of heterogeneity in the subgroups. 


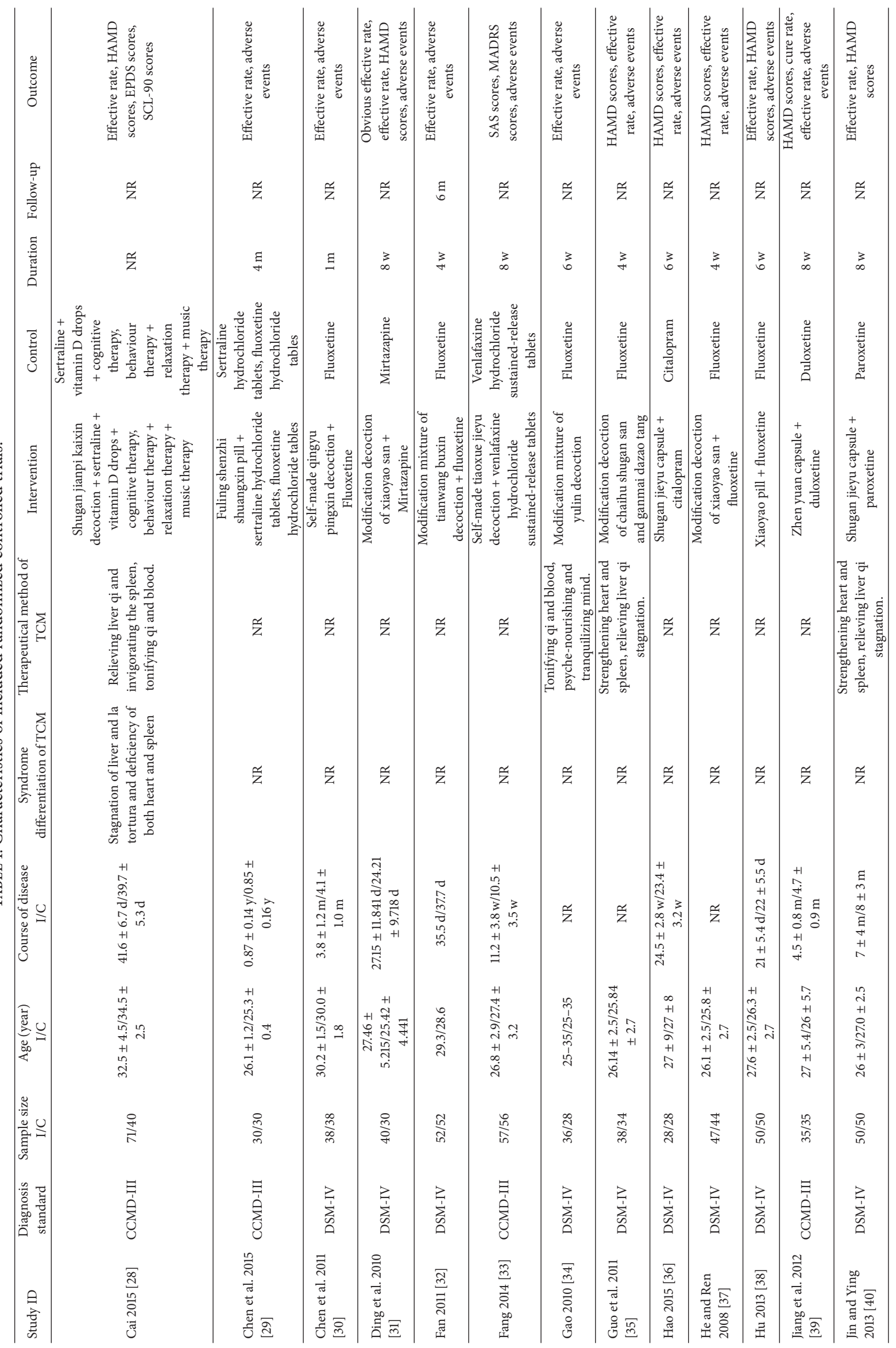




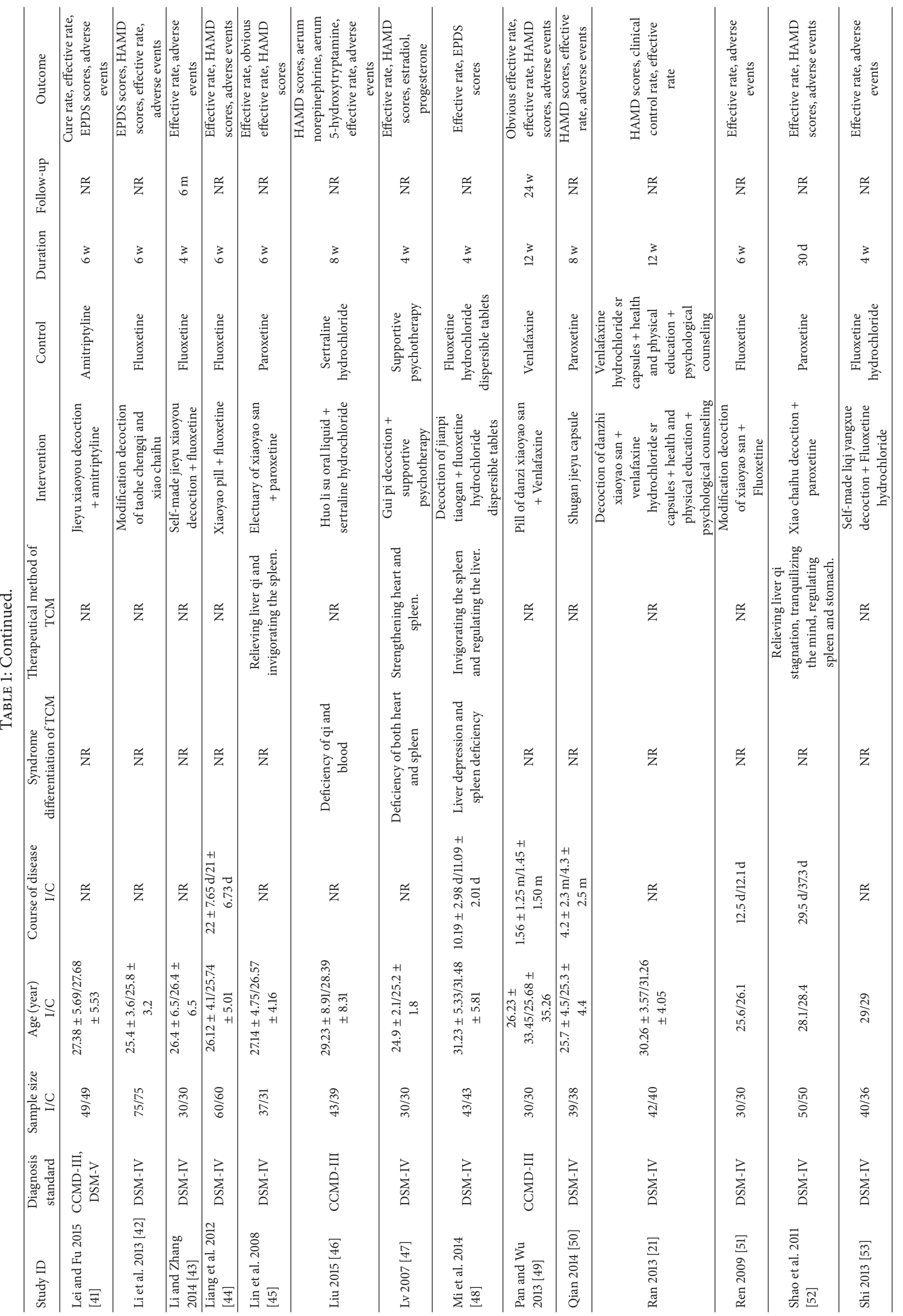




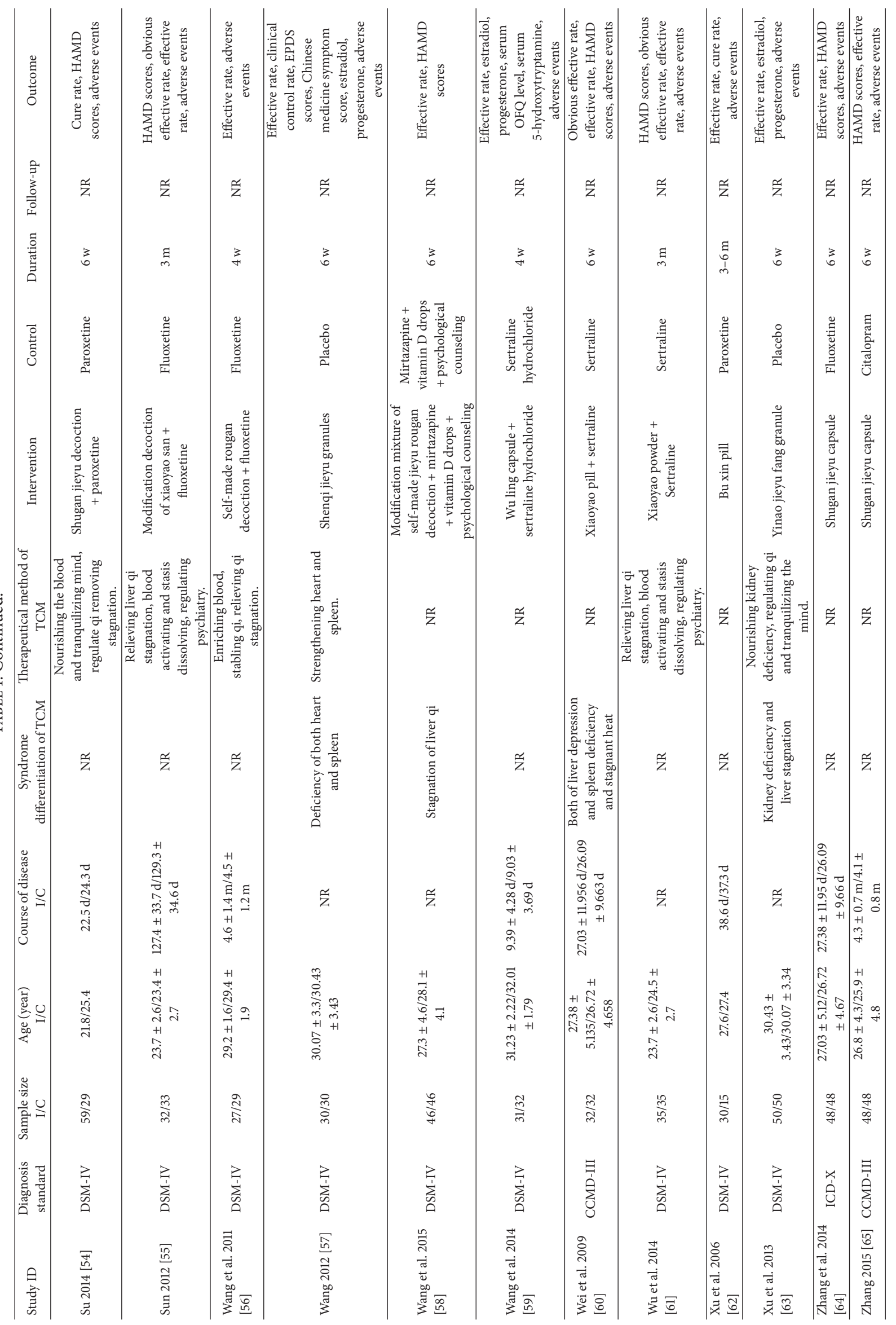




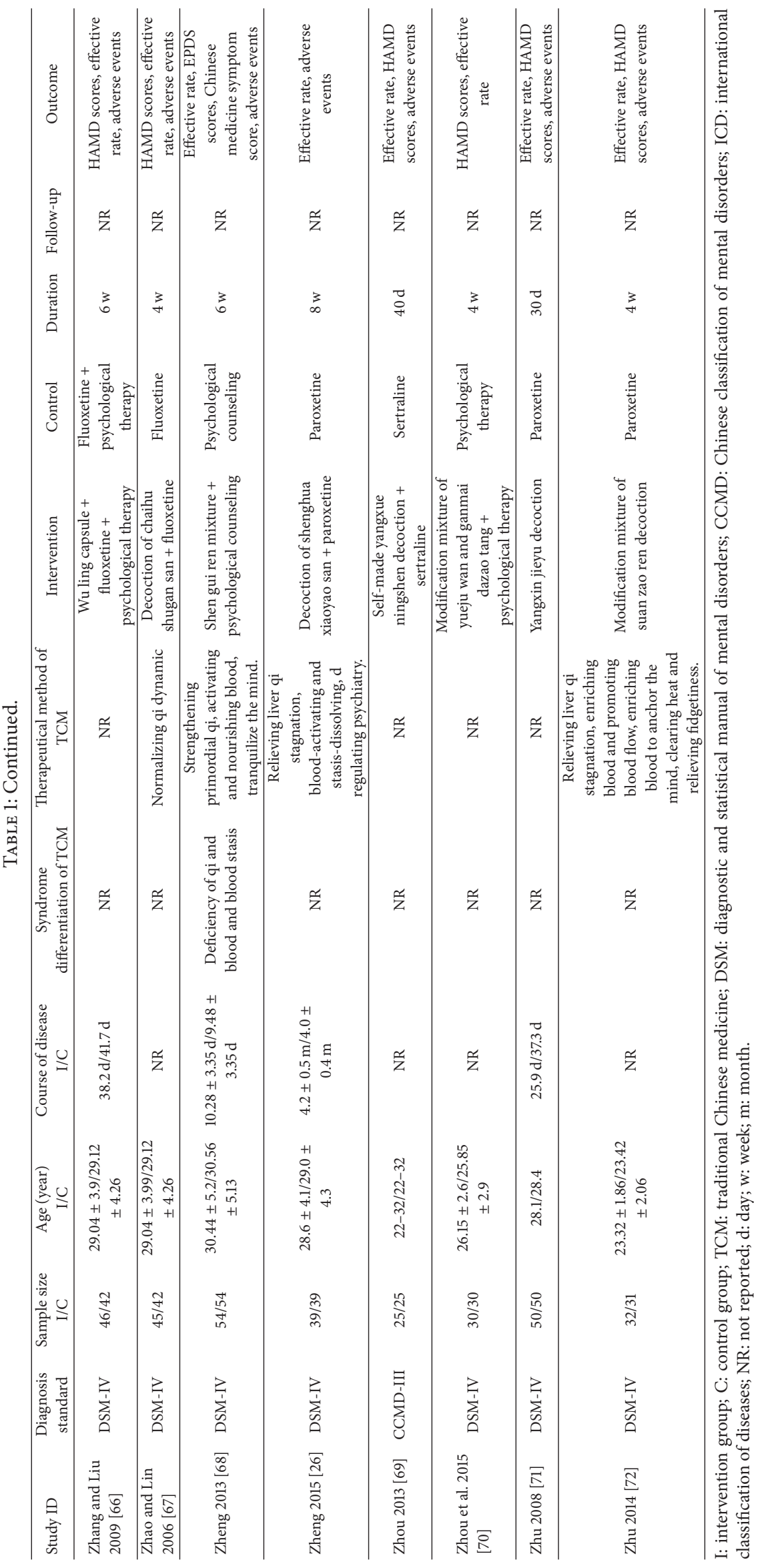


TABLE 2: Composition of formula and categories of TCM therapeutic methods.

\begin{tabular}{|c|c|c|c|}
\hline Study ID & $\begin{array}{l}\text { Categories of TCM } \\
\text { therapeutical } \\
\text { methods }\end{array}$ & Formula & Composition of formula \\
\hline Cai 2015 [28] & MRLQS + MSHS & $\begin{array}{l}\text { Shugan jianpi } \\
\text { kaixin decoction }\end{array}$ & $\begin{array}{l}\text { Radix bupleuri, Rhizoma chuanxiong, Rhizoma cyperi rotundi, } \\
\text { Pericarpium citri reticulatae, Radix paeoniae alba, Radix astragali, } \\
\text { Rhizoma atractylodes macrocephalae, Radix angelicae sinensis, Radix } \\
\text { polygalae tenuifoliae, Semen ziziphi spinosae, Fructus jujubae. }\end{array}$ \\
\hline $\begin{array}{l}\text { Chen et al. } \\
2011[30]\end{array}$ & MRLQS & $\begin{array}{l}\text { Self-made qingyu } \\
\text { pingxin decoction }\end{array}$ & $\begin{array}{l}\text { Massa medica fermentata, Radix bupleuri, Rhizoma cyperi rotundi, } \\
\text { Rhizoma atractylodes macrocephalae, Radix paeoniae alba, Radix } \\
\text { rehmanniae, Tu ophiopogonis japonici, Radix et rhizoma rhei, Radix } \\
\text { glycyrrhizae. }\end{array}$ \\
\hline $\begin{array}{l}\text { Chen et al. } \\
2015 \text { [29] }\end{array}$ & MRLQS + MSHS & $\begin{array}{l}\text { Fuling shenzhi } \\
\text { shuangxin pill }\end{array}$ & $\begin{array}{l}\text { Poria, Sclerotium pararadicis poriae cocos, Cinnabaris, Radix polygalae } \\
\text { tenuifoliae, Radix panacis ginseng, Semen ziziphi spinosae, Radix bupleuri, } \\
\text { Radix glycyrrhizae. }\end{array}$ \\
\hline $\begin{array}{l}\text { Ding et al. } \\
2010[31]\end{array}$ & MRLQS & $\begin{array}{l}\text { Modification } \\
\text { decoction of } \\
\text { xiaoyao san }\end{array}$ & $\begin{array}{l}\text { Radix bupleuri, Radix paeoniae alba, Radix angelicae sinensis, Rhizoma } \\
\text { atractylodes macrocephalae, Poria, Tuber curcumae, cortex albizziae } \\
\text { julibrissinis, Radix polygalae tenuifoliae, Semen ziziphi spinosae, Rhizoma } \\
\text { cyperi rotundi, Radix glycyrrhizae. }\end{array}$ \\
\hline Fan 2011 [32] & MRLQS + MTQB & $\begin{array}{l}\text { Modification } \\
\text { mixture of } \\
\text { tianwang buxin } \\
\text { decoction }\end{array}$ & $\begin{array}{l}\text { Tuber curcumae, Fructus schisandrae chinensis, Semen biotae orientalis, } \\
\text { Radix salviae miltiorrhizae, Radix paeoniae alba, Radix rehmanniae, Radix } \\
\text { polygalae tenuifoliae, Radix platycodi grandiflori, Radix bupleuri, } \\
\text { Cucurbita pepo, Semen ziziphi spinosae, Radix codonopsitis pilosulae, } \\
\text { Poria, Radix angelicae sinensis. }\end{array}$ \\
\hline $\begin{array}{l}\text { Fang } 2014 \\
{[33]}\end{array}$ & MRLQS + MBASD & $\begin{array}{l}\text { Self-made tiaoxue } \\
\text { jieyu decoction }\end{array}$ & $\begin{array}{l}\text { Radix bupleuri, Radix angelicae sinensis, Radix paeoniae alba, Poria, } \\
\text { Cortex magnoliae officinalis, Rhizoma cyperi rotundi, Tuber curcumae, } \\
\text { Cortex albizziae julibrissinis, Semen nelumbinis nucifarae, Semen ziziphi } \\
\text { spinosae, Herba leonuri, Radix pseudoginseng, Fructus crataegi, Herba } \\
\text { lophatheri, Radix polygalae tenuifoliae, Rhizoma acori tatarinowii, Radix } \\
\text { glycyrrhizae. }\end{array}$ \\
\hline Gao 2010 [34] & MTQB & $\begin{array}{l}\text { Modification } \\
\text { mixture of yulin } \\
\text { decoction }\end{array}$ & $\begin{array}{l}\text { Cornu degelatinum cervi, Semen cuscutae chinensis, Radix rehmanniae, } \\
\text { Radix angelicae sinensis, Radix paeoniae alba, Rhizoma chuanxiong, Radix } \\
\text { codonopsitis pilosulae, Rhizoma atractylodes macrocephalae, Poria, Cortex } \\
\text { eucommiae, Radix morindae officinalis, Rhizoma cyperi rotundi, Radix } \\
\text { glycyrrhizae. }\end{array}$ \\
\hline $\begin{array}{l}\text { Guo et al. } \\
2011[35]\end{array}$ & MRLQS + MSHS & $\begin{array}{l}\text { Modification } \\
\text { decoction of } \\
\text { chaihu shugan san } \\
\text { and ganmai dazao } \\
\text { tang }\end{array}$ & $\begin{array}{l}\text { Radix bupleuri, Fructus citri aurantii, Rhizoma cyperi rotundi, } \\
\text { Pericarpium citri reticulatae, Radix paeoniae alba, Rhizoma chuanxiong, } \\
\text { Radix glycyrrhizae, Fructus levis tritici aestiva, Fructus jujubae. }\end{array}$ \\
\hline Hao 2015 [36] & MRLQS + MSHS & $\begin{array}{l}\text { Shugan jieyu } \\
\text { capsule }\end{array}$ & Hypericum perforatum L., Acanthopanax senticosus. \\
\hline $\begin{array}{l}\text { He and Ren } \\
2008[37]\end{array}$ & MRLQS & $\begin{array}{l}\text { Modification } \\
\text { decoction of } \\
\text { xiaoyao san }\end{array}$ & $\begin{array}{l}\text { Radix bupleuri, Poria, Rhizoma atractylodis macrocephalae, Rhizoma } \\
\text { cyperi rotundi, Radix rehmanniae, Radix paeoniae alba, Radix et rhizoma } \\
\text { rhei, Radix glycyrrhizae. }\end{array}$ \\
\hline Hu 2013 [38] & MRLQS & Xiaoyao pill & $\begin{array}{l}\text { Radix angelicae sinensis, Radix paeoniae alba, Rhizoma atractylodes } \\
\text { macrocephalae, Radix bupleuri, Poria, and so forth. }\end{array}$ \\
\hline $\begin{array}{l}\text { Jiang et al. } \\
2012[39]\end{array}$ & MTQB & Zhen yuan capsule & Main flavour components is ginseng fruit saponins. \\
\hline $\begin{array}{l}\text { Jin and Ying } \\
2013[40]\end{array}$ & MRLQS + MSHS & $\begin{array}{l}\text { Shugan jieyu } \\
\text { capsule }\end{array}$ & Hypericum perforatum L, Acanthopanax senticosus. \\
\hline $\begin{array}{l}\text { Lei and Fu } \\
2015 \text { [41] }\end{array}$ & MRLQS + MSHS & $\begin{array}{l}\text { Jieyu xiaoyou } \\
\text { decoction }\end{array}$ & $\begin{array}{l}\text { Radix bupleuri, Rhizoma cyperi rotundi, Radix paeoniae alba, Radix } \\
\text { panacis ginseng, Poria, Sclerotium pararadicis poriae cocos, Radix } \\
\text { angelicae sinensis, Rhizoma atractylodes macrocephalae, Radix polygalae } \\
\text { tenuifoliae, Radix glycyrrhizae, Tu ophiopogonis japonici, Tuber curcumae. }\end{array}$ \\
\hline
\end{tabular}


TABLE 2: Continued.

\begin{tabular}{|c|c|c|c|}
\hline Study ID & $\begin{array}{l}\text { Categories of TCM } \\
\text { therapeutical } \\
\text { methods }\end{array}$ & Formula & Composition of formula \\
\hline $\begin{array}{l}\text { Li et al. } 2013 \\
{[42]}\end{array}$ & $\begin{array}{l}\text { MRLQS + MSHS + } \\
\quad \text { MBASD }\end{array}$ & $\begin{array}{l}\text { Modification } \\
\text { decoction of taohe } \\
\text { chengqi and xiao } \\
\text { chaihu }\end{array}$ & $\begin{array}{l}\text { Semen pruni persicae, Radix cinnamomi cassiae, Rheum officinale Baill, } \\
\text { Radix bupleuri, Radix scutellariae baicalensis, Pinellia ternata, Rhizoma } \\
\text { cyperi rotundi, Radix panacis ginseng, Kadsura interior, Ficus } \\
\text { simplicissima lour, Tuber curcumae, Os draconis, Semen ziziphi spinosae, } \\
\text { Radix glycyrrhizae. }\end{array}$ \\
\hline $\begin{array}{l}\text { Li and Zhang } \\
2014[43]\end{array}$ & MRLQS & $\begin{array}{l}\text { Self-made jieyu } \\
\text { xiaoyou decoction }\end{array}$ & $\begin{array}{l}\text { Radix bupleuri, Poria, Radix paeoniae alba, Rhizoma atractylodes } \\
\text { macrocephalae, Radix angelicae sinensis, Rhizoma cyperi rotundi, Radix } \\
\text { polygalae tenuifoliae, Tuber curcumae, Tu ophiopogonis japonici. }\end{array}$ \\
\hline $\begin{array}{l}\text { Liang et al. } \\
2012[44]\end{array}$ & MRLQS & Xiaoyao pill & $\begin{array}{l}\text { Radix angelicae sinensis, Radix paeoniae alba, Radix bupleuri, Rhizoma } \\
\text { atractylodes macrocephalae, Radix glycyrrhizae, Poria, Rhizoma zingiberis } \\
\text { preparatum, Peppermint. }\end{array}$ \\
\hline $\begin{array}{l}\text { Lin et al. } \\
2008[45]\end{array}$ & MRLQS & $\begin{array}{l}\text { Electuary of } \\
\text { xiaoyao san }\end{array}$ & $\begin{array}{l}\text { Radix bupleuri, Radix paeoniae alba, Radix angelicae sinensis, Poria, } \\
\text { Rhizoma atractylodes macrocephalae, Radix glycyrrhizae, Peppermint, } \\
\text { Uncooked rhizoma zingiberis. }\end{array}$ \\
\hline Liu 2015 [46] & MTQB & $\begin{array}{l}\text { Huo li su oral } \\
\text { liquid }\end{array}$ & $\begin{array}{l}\text { Radix astragali, Rhizoma polygonati, Fructus lycii chinensis, Radix } \\
\text { polygoni multiflori, Radix salviae miltiorrhizae, Herba epimedii. }\end{array}$ \\
\hline Lv 2007 [47] & MSHS & Gui pi decoction & $\begin{array}{l}\text { Radix astragali, Arillus longan, Radix panacis ginseng, Rhizoma } \\
\text { atractylodes macrocephalae, Radix angelicae sinensis, Sclerotium } \\
\text { pararadicis poriae cocos, Semen ziziphi spinosae, Radix polygalae } \\
\text { tenuifolia, Radix aucklandiae, Radix glycyrrhizae. }\end{array}$ \\
\hline $\begin{array}{l}\text { Mi et al. } 2014 \\
{[48]}\end{array}$ & MRLQS + MSHS & $\begin{array}{l}\text { Decoction of jianpi } \\
\text { tiaogan }\end{array}$ & $\begin{array}{l}\text { Radix astragali, Radix angelicae sinensis, Radix paeoniae alba, Radix } \\
\text { bupleuri, Poria, Rhizoma atractylodes macrocephalae, Rhizoma pinelliae } \\
\text { ternatae, Cortex magnoliae officinalis, Rhizoma polygonati, Uncooked } \\
\text { rhizoma zingiberis, Caulis perillae, Radix glycyrrhizae. }\end{array}$ \\
\hline $\begin{array}{l}\text { Pan and } \mathrm{Wu} \\
2013[49]\end{array}$ & MRLQS & Danzi xiaoyao pill & $\begin{array}{l}\text { Radix bupleuri, Radix angelicae sinensis, Radix paeoniae alba, Poria, } \\
\text { Rhizoma atractylodes macrocephala, Cortex radix moutan, Fructus } \\
\text { gardeniae jasminoidis, Peppermint, Radix glycyrrhizae. }\end{array}$ \\
\hline $\begin{array}{l}\text { Qian } 2014 \\
{[50]}\end{array}$ & MRLQS + MSHS & $\begin{array}{l}\text { Shugan jieyu } \\
\text { capsule }\end{array}$ & Hypericum perforatum L, Acanthopanax senticosus. \\
\hline Ran 2013 [21] & MRLQS & $\begin{array}{c}\text { Decoction of } \\
\text { danzhi xiaoyao san }\end{array}$ & $\begin{array}{l}\text { Radix bupleuri, Peppermint, Cortex radix moutan, Fructus gardenige, } \\
\text { Radix angelicae sinensis, Radix paeoniae alba, Rhizoma atractylodes } \\
\text { macrocephalae, Poria, Radix glycyrrhizae. }\end{array}$ \\
\hline Ren 2009 [51] & MRLQS & $\begin{array}{l}\text { Modification } \\
\text { decoction of } \\
\text { xiaoyao san }\end{array}$ & $\begin{array}{l}\text { Radix bupleuri, Poria, Radix paeoniae alba, Rhizoma atractylodes } \\
\text { macrocephalae, Radix angelicae sinensis, Radix et rhizoma rhei, Rhizoma } \\
\text { cyperi rotundi, Tu ophiopogonis japonici, Radix rehmanniae. }\end{array}$ \\
\hline $\begin{array}{l}\text { Shao et al. } \\
2011 \text { [52] }\end{array}$ & MRLQS & $\begin{array}{l}\text { Xiao chaihu } \\
\text { decoction }\end{array}$ & $\begin{array}{l}\text { Radix bupleuri, Radix scutellariae baicalensis, Rhizoma pinelliae ternatae, } \\
\text { Fructus jujubae, Radix glycyrrhizae, Uncooked rhizoma zingiberis, Radix } \\
\text { paeoniae alba, Rhizoma atractylodes macrocephalae, Bulbus lilii, Semen } \\
\text { ziziphi spinosae. }\end{array}$ \\
\hline Shi 2013 [53] & MRLQS + MSHS & $\begin{array}{c}\text { Self-made liqi } \\
\text { yangxue decoction }\end{array}$ & $\begin{array}{l}\text { Radix bupleuri, Radix angelicae sinensis, Fructus citri aurantii, Rhizoma } \\
\text { cyperi rotundi, Pericarpium citri reticulatae, Tuber curcumae, Radix } \\
\text { paeoniae alba, Radix astragali, Radix codonopsitis pilosulae, Fructus } \\
\text { jujubae, Arillus longan, Sclerotium pararadicis poriae cocos, Semen biotae } \\
\text { orientalis, Radix polygalae tenuifoliae, Semen nelumbinis nucifarae, Radix } \\
\text { glycyrrhizae. }\end{array}$ \\
\hline Su 2014 [54] & MRLQS + MBASD & $\begin{array}{c}\text { Shugan jieyu } \\
\text { decoction }\end{array}$ & $\begin{array}{l}\text { Radix bupleuri, Radix angelicae sinensis, Radix paeoniae alba, Tuber } \\
\text { curcumae, Poria, Rhizoma chuanxiong, Rhizoma cyperi rotundi, cortex } \\
\text { albizziae julibrissinis, Flos carthami tinctorii, Semen pruni persicae, Radix } \\
\text { rehmanniae, Bulbus lilii. }\end{array}$ \\
\hline Sun 2012 [55] & MRLQS & $\begin{array}{l}\text { Modification } \\
\text { decoction of } \\
\text { xiaoyao san }\end{array}$ & $\begin{array}{l}\text { Radix bupleuri, Poria, Radix paeoniae alba, Rhizoma atractylodes } \\
\text { macrocephalae, Radix angelicae sinensis, Semen ziziphi spinosae, Rhizoma } \\
\text { chuanxiong, Radix glycyrrhizae. }\end{array}$ \\
\hline
\end{tabular}


TABLe 2: Continued.

\begin{tabular}{|c|c|c|c|}
\hline Study ID & $\begin{array}{l}\text { Categories of TCM } \\
\text { therapeutical } \\
\text { methods }\end{array}$ & Formula & Composition of formula \\
\hline $\begin{array}{l}\text { Wang et al. } \\
2011[56]\end{array}$ & MRLQS & $\begin{array}{l}\text { Self-made rougan } \\
\text { decoction }\end{array}$ & $\begin{array}{l}\text { Radix bupleuri, Radix angelicae sinensis, Rhizoma atractylodes } \\
\text { macrocephalae, Radix paeoniae alba, Tuber curcumae, Citrus reticulata } \\
\text { blanco, Radix rehmanniae, Tu ophiopogonis japonici, Radix glycyrrhizae. }\end{array}$ \\
\hline $\begin{array}{l}\text { Wang } 2012 \\
{[57]}\end{array}$ & MSHS & $\begin{array}{l}\text { Shenqi jieyu } \\
\text { granules }\end{array}$ & $\begin{array}{l}\text { Radix astragali, Radix codonopsitis pilosulae, Radix angelicae sinensis, } \\
\text { Semen ziziphi spinosae, Fructus corni, and so forth. }\end{array}$ \\
\hline $\begin{array}{l}\text { Wang et al. } \\
2014[59]\end{array}$ & MTQB & Wu ling capsule & Main flavour components is Xylaria nigripes powder. \\
\hline $\begin{array}{l}\text { Wang et al. } \\
2015[58]\end{array}$ & MRLQS & $\begin{array}{l}\text { Modification } \\
\text { mixture of } \\
\text { self-made jieyu } \\
\text { rougan decoction }\end{array}$ & $\begin{array}{l}\text { Radix bupleuri, Fructus citri aurantii, Rhizoma cyperi rotundi, } \\
\text { Pericarpium citri reticulatae, Radix angelicae sinensis, Radix paeoniae alba, } \\
\text { Cortex albizziae julibrissinis, Tuber fleeceflower stem, Semen ziziphi } \\
\text { spinosae, Rhizoma atractylodes macrocephalae, Poria, Uncooked rhizoma } \\
\text { zingiberis, Radix glycyrrhizae. }\end{array}$ \\
\hline $\begin{array}{l}\text { Wei et al. } \\
2009[60]\end{array}$ & MRLQS & Xiaoyao pill & $\begin{array}{l}\text { Radix bupleuri, Radix angelicae sinensis, Radix paeoniae alba, Poria, } \\
\text { Rhizoma atractylodis macrocephalae, Radix clycyrrhizae, and so forth. }\end{array}$ \\
\hline $\begin{array}{l}\text { Wu et al. } 2014 \\
{[61]}\end{array}$ & MRLQS & Xiaoyao powder & $\begin{array}{l}\text { Radix bupleuri, Poria, Radix paeoniae alba, Rhizoma atractylodes } \\
\text { macrocephalae, Radix angelicae sinensis, Semen ziziphi spinosae, Rhizoma } \\
\text { chuanxiong, Radix glycyrrhizae. }\end{array}$ \\
\hline $\begin{array}{l}\text { Xu et al. } 2006 \\
{[62]}\end{array}$ & MTQB & Bu xin pill & $\begin{array}{l}\text { Radix angelicae sinensis, Rhizoma chuanxiong, Radix paeoniae alba, Radix } \\
\text { rehmanniae, Radix panacis ginseng, Radix scrophulariae ningpoensis, Tu } \\
\text { ophiopogonis japonici, Radix asparagi, Cinnabaris, Poria, Radix polygalae } \\
\text { tenuifoliae, Semen ziziphi spinosae, Semen biotae orientalis. }\end{array}$ \\
\hline $\begin{array}{l}\text { Xu et al. } 2013 \\
{[63]}\end{array}$ & MRLQS + MTQB & $\begin{array}{l}\text { Yinao jieyu fang } \\
\text { granule }\end{array}$ & Acanthopanax senticosus, Tuber curcumae, and so forth. \\
\hline $\begin{array}{l}\text { Zhang and } \\
\text { Liu } 2009 \text { [66] }\end{array}$ & MTQB & Wu ling capsule & Main flavour components is Xylaria nigripes powder. \\
\hline $\begin{array}{l}\text { Zhang et al. } \\
2014[64]\end{array}$ & MRLQS + MSHS & $\begin{array}{l}\text { Shugan jieyu } \\
\text { capsule }\end{array}$ & Hypericum perforatum L, Acanthopanax senticosus. \\
\hline $\begin{array}{l}\text { Zhang } 2015 \\
{[65]}\end{array}$ & MRLQS + MSHS & $\begin{array}{l}\text { Shugan jieyu } \\
\text { capsule }\end{array}$ & Hypericum perforatum L, Acanthopanax senticosus. \\
\hline $\begin{array}{l}\text { Zhao and Lin } \\
2006 \text { [67] }\end{array}$ & MRLQS & $\begin{array}{c}\text { Decoction of } \\
\text { chaihu shugan san }\end{array}$ & $\begin{array}{l}\text { Radix bupleuri, Fructus citri aurantii, Rhizoma cyperi rotundi, } \\
\text { Pericarpium citri reticulatae, Radix paeoniae alba, Rhizoma chuanxiong, } \\
\text { Radix glycyrrhizae. }\end{array}$ \\
\hline $\begin{array}{l}\text { Zheng } 2013 \\
{[68]}\end{array}$ & MTQB + MBASD & $\begin{array}{c}\text { Shen gui ren } \\
\text { mixture }\end{array}$ & Radix panacis ginseng, Radix angelicae sinensis, Semen ziziphi spinosae. \\
\hline $\begin{array}{l}\text { Zheng } 2015 \\
\text { [26] }\end{array}$ & MRLQS + MBASD & $\begin{array}{l}\text { Decoction of } \\
\text { shenghua xiaoyao } \\
\text { san }\end{array}$ & $\begin{array}{l}\text { Radix angelicae sinensis, Radix rubrus paeoniae lactiflorae, Poria, Rhizoma } \\
\text { atractylodes macrocephalae, Semen pruni persicae, Radix bupleuri, } \\
\text { Rhizoma zingiberis preparatum, Peppermint, Radix glycyrrhizae. }\end{array}$ \\
\hline $\begin{array}{l}\text { Zhou } 2013 \\
{[69]}\end{array}$ & MRLQS + MTQB & $\begin{array}{l}\text { Self-made yangxue } \\
\text { ningshen } \\
\text { decoction }\end{array}$ & $\begin{array}{l}\text { Radix angelicae sinensis, Radix paeoniae alba, Radix bupleuri, Radix } \\
\text { salviae miltiorrhizae, Tuber curcumae, Semen ziziphi spinosae, cortex } \\
\text { albizziae julibrissinis, Radix polygalae tenuifoliae, Radix glycyrrhizae. }\end{array}$ \\
\hline $\begin{array}{l}\text { Zhou et al. } \\
2015 \text { [70] }\end{array}$ & MRLQS + MSHS & $\begin{array}{l}\text { Modification } \\
\text { mixture of yueju } \\
\text { wan and ganmai } \\
\text { dazao tang }\end{array}$ & $\begin{array}{l}\text { Rhizoma cyperi rotundi, Rhizoma chuanxion, Rhizoma atractylodis, Massa } \\
\text { medica fermentata, Fructus gardeniae jasminoidis, Radix glycyrrhizae, } \\
\text { Fructus levis tritici aestiva, Fructus jujubae. }\end{array}$ \\
\hline Zhu 2008 [71] & MRLQS + MTQB & $\begin{array}{l}\text { Yangxin jieyu } \\
\text { decoction }\end{array}$ & $\begin{array}{l}\text { Radix astragali, Radix bupleuri, Radix scutellariae baicalensis, Radix } \\
\text { panacis ginseng, Radix glycyrrhizae, Radix rehmanniae, Fructus } \\
\text { schisandrae chinensis, Radix angelicae sinensis, Semen biotae orientalis, } \\
\text { Semen ziziphi spinosae, Radix salviae miltiorrhizae, Uncooked rhizoma } \\
\text { zingiberis, Fructus jujubae. }\end{array}$ \\
\hline Zhu 2014 [72] & $\begin{array}{c}\text { MRLQS + MTQB } \\
+ \text { MBASD }\end{array}$ & $\begin{array}{l}\text { Modification } \\
\text { mixture of suan } \\
\text { zao ren decoction }\end{array}$ & $\begin{array}{l}\text { Semen ziziphi spinosae, Poria, Rhizoma chuanxiong, Rhizoma } \\
\text { anemarrhenae, Tuber curcumae, Radix angelicae sinensis, Radix } \\
\text { glycyrrhizae, Radix bupleuri. }\end{array}$ \\
\hline
\end{tabular}




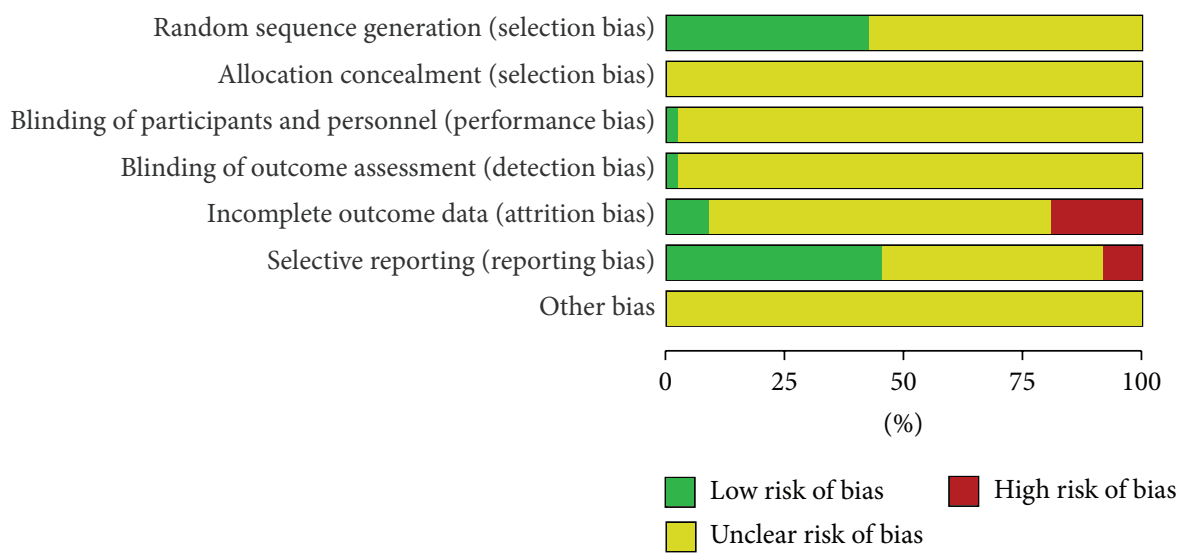

FIGURE 2: Risk of bias graph: review authors' judgments about each risk of bias item presented as percentages across all included studies.

3.3.1. HAMD Scores. 30 trials $[21,28,31,35-40,42,44-$ $47,49,50,52,54,55,58,60,61,64-67,69-72]$ reported outcomes in HAMD score after treatment. Seven trials [35, $42,50,64,65,71,72$ ] which compared CHM with routine treatments showed statistical heterogeneity in the consistency of the results $\left(I^{2}=95 \%\right)$, and a random effects model was used for meta-analysis. The pooled results showed that there were statistically significant differences between CHM and routine treatments ( $\mathrm{MD}-2.60,95 \% \mathrm{CI}-4.55$ to -0.64 ) in reducing HAMD score. Subgroup analysis showed that MRLQS plus MSHS (4 trials) [35, 50, 64, 65] had a superior effect to routine treatments (MD $-1.91,95 \% \mathrm{CI}-3.37$ to -0.44). MRLQS plus MTQB (1 trial) [71] and MRLQS plus MSHS plus MBASD (1 trial) [42] had a statistically significant reduction in HAMD score as compared to routine treatments. Meanwhile, one trial [72] reported no significant difference between MRLQS plus MTQB plus MBASD and routine treatments. To compare the effects of $\mathrm{CHM}$ plus routine treatments with routine treatments alone, 23 trials [21, 28, $31,36-40,44-47,49,52,54,55,58,60,61,66,67,69,70]$ were evaluated. Heterogeneity testing of these trials indicated a significant difference $\left(I^{2}=85 \%\right)$; therefore, a random effects model was used. The pooled results showed that there were statistically significant differences between CHM plus routine treatments and routine treatments alone ( $\mathrm{MD}-3.00,95 \%$ CI -3.73 to -2.26$)$ in reducing HAMD scores. Subgroup analysis was conducted on the Chinese medicine therapeutic methods of MRLQS, MTQB, MSHS, MRLQS plus MSHS, MRLQS plus MBASD, and MRLQS plus MTQB. Pooled results from 13 trials $[21,31,37,38,44,45,49,52,55,58$, $60,61,67]$ showed that MRLQS combined with routine treatments had superior effectiveness as compared to routine treatments alone (MD -3.19, 95\% CI -4.41 to -1.97 ) in reducing HAMD score. Three trials $[39,46,66]$ indicated that MTQB combined with routine treatments was more effective than routine treatments alone ( $\mathrm{MD}-2.67,95 \% \mathrm{CI}-4.48$ to $-0.87)$. Four trials $[28,36,40,70]$ demonstrated that MRLQS plus MSHS combined with routine treatments had superior effectiveness as compared to routine treatments alone (MD $-2.67,95 \%$ CI -3.20 to -2.14 ). As shown in Table 3, the results demonstrated that MSHS combined with routine treatments (1 trial) [47] or MRLQS plus MBASD combined with routine treatments (1 trial) [54] was more effective than routine treatments alone in reducing HAMD score. One trial [69] demonstrated no statistically significant differences between MRLQS plus MTQB combined with routine treatments and routine treatments alone (shown in Table 3).

3.3.2. EPDS Scores. Six trials [28, 41, 42, 48, 57, 68] provided data on EPDS score changes after treatment. Only one trial [57] compared CHM using MSHS with a placebo. The study results showed that MSHS was more effective than the placebo (MD - 2.67, 95\% CI -3.88 to -1.46) in reducing EPDS score. Two trials $[42,68]$ which compared CHM with routine treatments conducted a random effects model for metaanalysis because of statistical heterogeneity of the results $\left(I^{2}=\right.$ $90 \%)$. The pooled results showed significant beneficial effects of $\mathrm{CHM}$ as compared to routine treatments ( $\mathrm{MD}-3.36$, $95 \%$ CI -6.05 to -0.66 ). Subgroup analysis in these trials revealed that the use of MTQB plus MBASD (1 trial) [68] or MRLQS plus MSHS plus MBASD (1 trial) [42] resulted in a statistically significant reduction in EPDS score when compared with routine treatments alone. Three trials [28, 41, 48] compared CHM combined with routine treatments with routine treatments alone. These trials used MRLQS plus MSHS as the therapeutic approaches of CHM and used a random effects model for pooled analysis because of statistical heterogeneity $\left(I^{2}=75 \%\right)$. The pooled result showed that CHM (MRLQS plus MSHS) combined with routine treatments had superior effectiveness when compared to routine treatments alone ( $\mathrm{MD}-3.80,95 \% \mathrm{CI}-5.27$ to -2.34 ) in reducing EPDS score (shown in Table 3).

3.3.3. Serum Estradiol. Four trials $[47,57,59,63]$ reported the effect on serum estradiol of CHM alone or in combination with routine treatments. Two trials $[57,63]$ compared the effect of CHM with a placebo and used a random effects model for meta-analysis because of statistical heterogeneity $\left(I^{2}=88 \%\right)$. The pooled result showed no statistically significant differences between the groups (MD 29.01, 95\% CI -2.54 
TABLE 3: Effect estimates of CHM for PPD.

\begin{tabular}{|c|c|c|c|}
\hline Outcome or subgroup & Trials & Effect estimates [95\% CI] & $P$ value \\
\hline \multicolumn{4}{|l|}{ (1) HAMD scores } \\
\hline \multicolumn{4}{|l|}{ (1.1) $\mathrm{CHM}$ versus routine treatments } \\
\hline MRLQS + MSHS versus routine treatments & 4 & $\mathrm{MD}-1.91[-3.37,-0.44]$ & 0.01 \\
\hline MRLQS + MTQB versus routine treatments & 1 & $\mathrm{MD}-7.30[-8.23,-6.37]$ & $<0.00001$ \\
\hline MRLQS + MSHS + MBASD versus routine treatments & 1 & $\mathrm{MD}-2.93[-4.36,-1.50]$ & $<0.0001$ \\
\hline MRLQS + MTQB + MBASD versus routine treatments & 1 & $\mathrm{MD}-0.22[-2.40,1.96]$ & 0.84 \\
\hline Meta-analysis $[R E M]$ (heterogeneity: $I^{2}=95 \%, P<0.00001$ ) & 7 & $\mathrm{MD}-2.60[-4.55,-0.64]$ & 0.009 \\
\hline \multicolumn{4}{|l|}{ (1.2) $C H M+$ routine treatments versus routine treatments } \\
\hline MRLQS + routine treatments versus routine treatments & 13 & $\mathrm{MD}-3.19[-4.41,-1.97]$ & $<0.00001$ \\
\hline MSHS + routine treatments versus routine treatments & 1 & $\mathrm{MD}-4.10[-6.23,-1.97]$ & 0.0002 \\
\hline $\mathrm{MTQB}$ + routine treatments versus routine treatments & 3 & $\mathrm{MD}-2.67[-4.48,-0.87]$ & 0.004 \\
\hline MRLQS + MSHS + routine treatments versus routine treatments & 4 & $\mathrm{MD}-2.67[-3.20,-2.14]$ & $<0.00001$ \\
\hline MRLQS + MBASD + routine treatments versus routine treatments & 1 & $\mathrm{MD}-4.10[-6.65,-1.55]$ & 0.002 \\
\hline MRLQS + MTQB + routine treatments versus routine treatments & 1 & $\mathrm{MD}-1.74[-3.96,0.48]$ & 0.12 \\
\hline Meta-analysis $[R E M]$ (heterogeneity: $I^{2}=85 \%, P<0.00001$ ) & 23 & $\mathrm{MD}-3.00[-3.73,-2.26]$ & $<0.00001$ \\
\hline \multicolumn{4}{|l|}{ (2) EPDS scores } \\
\hline \multicolumn{4}{|l|}{ (2.1) $\mathrm{CHM}$ versus placebo } \\
\hline MSHS versus placebo & 1 & $\mathrm{MD}-2.67[-3.88,-1.46]$ & $<0.0001$ \\
\hline \multicolumn{4}{|l|}{ (2.2) $\mathrm{CHM}$ versus routine treatments } \\
\hline MTQB + MBASD versus routine treatments & 1 & $\mathrm{MD}-4.70[-5.76,-3.64]$ & $<0.00001$ \\
\hline MRLQS + MSHS + MBASD versus routine treatments & 1 & $\mathrm{MD}-1.95[-3.30,-0.60]$ & 0.005 \\
\hline Meta-analysis [REM] (heterogeneity: $I^{2}=90 \%, P=0.002$ ) & 2 & $\mathrm{MD}-3.36[-6.05,-0.66]$ & 0.01 \\
\hline \multicolumn{4}{|l|}{ (2.3) CHM + routine treatments versus routine treatments } \\
\hline MRLQS + MSHS + routine treatments versus routine treatments & 3 & $\mathrm{MD}-3.80[-5.27,-2.34]$ & $<0.00001$ \\
\hline Meta-analysis [REM] (heterogeneity: $I^{2}=75 \%, P=0.02$ ) & 3 & $\mathrm{MD}-3.80[-5.27,-2.34]$ & $<0.00001$ \\
\hline \multicolumn{4}{|l|}{ (3) Serum estradiol } \\
\hline \multicolumn{4}{|l|}{ (3.1) $\mathrm{CHM}$ versus placebo } \\
\hline MSHS versus placebo & 1 & MD $11.55[-8.58,31.68]$ & 0.26 \\
\hline MRLQS + MTQB versus placebo & 1 & MD $43.85[34.90,52.80]$ & $<0.00001$ \\
\hline Meta-analysis [REM] (heterogeneity: $I^{2}=88 \%, P=0.004$ ) & 2 & MD $29.01[-2.54,60.56]$ & 0.07 \\
\hline \multicolumn{4}{|l|}{ (3.2) $\mathrm{CHM}+$ routine treatments versus routine treatments } \\
\hline MSHS + routine treatments versus routine treatments & 1 & MD $26.73[5.06,48.40]$ & 0.02 \\
\hline $\mathrm{MTQB}+$ routine treatments versus routine treatments & 1 & MD $38.58[35.58,41.58]$ & $<0.00001$ \\
\hline Meta-analysis [FEM] (heterogeneity: $I^{2}=11 \%, P=0.29$ ) & 2 & MD $38.36[35.38,41.33]$ & $<0.00001$ \\
\hline \multicolumn{4}{|l|}{ (4) Progesterone } \\
\hline \multicolumn{4}{|l|}{ (4.1) $C H M$ versus placebo } \\
\hline MSHS versus placebo & 1 & $\mathrm{MD}-1.99[-7.28,3.30]$ & 0.46 \\
\hline MRLQS + MTQB versus placebo & 1 & $\mathrm{MD}-2.11[-3.10,-1.12]$ & $<0.0001$ \\
\hline Meta-analysis [FEM] (heterogeneity: $I^{2}=0 \%, P=0.97$ ) & 2 & $\mathrm{MD}-2.11[-3.08,-1.14]$ & $<0.0001$ \\
\hline \multicolumn{4}{|l|}{ (4.2) $\mathrm{CHM}+$ routine treatments versus routine treatments } \\
\hline MSHS + routine treatments versus routine treatments & 1 & $\mathrm{MD}-3.89[-8.92,1.14]$ & 0.13 \\
\hline MTQB + routine treatments versus routine treatments & 1 & MD -11.64 [-13.41, -9.87] & $<0.00001$ \\
\hline Meta-analysis [REM] (heterogeneity: $I^{2}=88 \%, P=0.004$ ) & 2 & $\mathrm{MD}-8.14[-15.70,-0.58]$ & 0.03 \\
\hline \multicolumn{4}{|l|}{ (5) Incidence of adverse events } \\
\hline \multicolumn{4}{|l|}{ (5.1) CHM versus placebo } \\
\hline MSHS versus placebo & 1 & RR $0.20[0.01,4.00]$ & 0.29 \\
\hline MRLQS + MTQB versus placebo & 1 & RR $3.00[0.13,71.92]$ & 0.50 \\
\hline Meta-analysis [FEM] (heterogeneity: $I^{2}=33 \%, P=0.22$ ) & 2 & RR $0.67[0.11,3.91]$ & 0.65 \\
\hline
\end{tabular}


TABLe 3: Continued.

\begin{tabular}{|c|c|c|c|}
\hline Outcome or subgroup & Trials & Effect estimates [95\% CI] & $P$ value \\
\hline \multicolumn{4}{|l|}{ (5.2) CHM versus routine treatments } \\
\hline MTQB versus routine treatments & 2 & RR $0.12[0.04,0.36]$ & 0.0001 \\
\hline MRLQS + MSHS versus routine treatments & 4 & RR $0.21[0.10,0.47]$ & 0.0001 \\
\hline MRLQS + MTQB versus routine treatments & 1 & RR $0.06[0.00,0.99]$ & 0.05 \\
\hline MRLQS + MSHS + MBASD versus routine treatments & 1 & RR $0.73[0.31,1.71]$ & 0.46 \\
\hline MRLQS + MTQB + MBASD versus routine treatments & 1 & RR $0.03[0.00,0.42]$ & 0.010 \\
\hline Meta-analysis [REM] (heterogeneity: $I^{2}=57 \%, P=0.02$ ) & 9 & RR $0.18[0.09,0.38]$ & $<0.00001$ \\
\hline \multicolumn{4}{|l|}{ (5.3) $\mathrm{CHM}+$ routine treatments versus routine treatments } \\
\hline MRLQS + routine treatments versus routine treatments & 9 & RR $0.45[0.32,0.64]$ & $<0.00001$ \\
\hline MTQB + routine treatments versus routine treatments & 3 & RR $0.76[0.31,1.84]$ & 0.54 \\
\hline MRLQS + MSHS + routine treatments versus routine treatments & 3 & RR $0.45[0.22,0.93]$ & 0.03 \\
\hline MRLQS + MTQB + routine treatments versus routine treatments & 2 & RR $0.32[0.09,1.14]$ & 0.08 \\
\hline MRLQS + MBASD + routine treatments versus routine treatments & 2 & RR $0.73[0.18,2.93]$ & 0.66 \\
\hline Meta-analysis [REM] (heterogeneity: $I^{2}=56 \%, P=0.002$ ) & 19 & RR $0.49[0.37,0.65]$ & $<0.00001$ \\
\hline \multicolumn{4}{|l|}{ (6) TESS } \\
\hline \multicolumn{4}{|l|}{ (6.1) $\mathrm{CHM}$ versus routine treatments } \\
\hline MRLQS + MSHS versus routine treatments & 1 & $\mathrm{MD}-2.05[-3.00,-1.10]$ & $<0.0001$ \\
\hline \multicolumn{4}{|l|}{ (6.2) $\mathrm{CHM}+$ routine treatments versus routine treatments } \\
\hline MRLQS + routine treatments versus routine treatments & 2 & $\mathrm{MD}-2.31[-3.12,-1.50]$ & $<0.00001$ \\
\hline $\mathrm{MTQB}+$ routine treatments versus routine treatments & 1 & $\mathrm{MD}-3.02[-4.25,-1.79]$ & $<0.00001$ \\
\hline MRLQS + MTQB + routine treatments versus routine treatments & 1 & $\mathrm{MD}-0.83[-1.50,-0.16]$ & 0.01 \\
\hline MRLQS + MBASD + routine treatments versus routine treatments & 1 & $\mathrm{MD}-1.40[-1.72,-1.08]$ & $<0.00001$ \\
\hline Meta-analysis [REM] (heterogeneity: $I^{2}=71 \%, P=0.008$ ) & 5 & $\mathrm{MD}-1.80[-2.46,-1.14]$ & $<0.00001$ \\
\hline \multicolumn{4}{|l|}{ (7) SERS } \\
\hline \multicolumn{4}{|l|}{ (7.1) $\mathrm{CHM}+$ routine treatments versus routine treatments } \\
\hline MRLQS + routine treatments versus routine treatments & 2 & $\mathrm{MD}-5.19[-9.73,-0.64]$ & 0.03 \\
\hline Meta-analysis [REM] (heterogeneity: $I^{2}=98 \%, P<0.00001$ ) & 2 & MD $5.19[-9.73,-0.64]$ & 0.03 \\
\hline
\end{tabular}

FEM: fixed effects model, REM: random effects model.

to 60.56) in increasing serum estradiol. Subgroup analysis showed that MRLQS plus MTQB (1 trial) [63] was more effective than routine treatments alone. No significant difference was observed in another trial [57] that compared MSHS with a placebo. In comparing CHM combined with routine treatments to routine treatments alone, the data from 2 trials [ 47 , 59] was pooled and a fixed effects model was used because of no statistical heterogeneity $\left(I^{2}=11 \%\right)$. The results revealed that $\mathrm{CHM}$ combined with routine treatments had superior effectiveness when compared with routine treatments alone (MD 38.36, 95\% CI 35.38 to 41.33). The subgroup analysis revealed that MSHS combined with routine treatments (1 trial) [47] or MTQB combined with routine treatments (1 trial) [59] was more effective than routine treatments alone in increasing serum estradiol (shown in Table 3 ).

3.3.4. Progesterone. Four trials [47, 57, 59, 63] reported progesterone levels in patients receiving $\mathrm{CHM}$ alone or in combination with routine treatments. Two trials $[57,63]$ compared effect of CHM to a placebo, and the data was pooled with a fixed effects model being used because of no statistical heterogeneity $\left(I^{2}=0 \%\right)$. The results showed that $\mathrm{CHM}$ was more effective than a placebo in decreasing progesterone (MD $-2.11,95 \% \mathrm{CI}-3.08$ to -1.14 ). The subgroup analysis showed that MRLQS plus MTQB (1 trial) [63] was more effective than routine treatments alone, and no significant differences were observed in another trial [57] when MSHS was compared to a placebo. In comparing CHM combined with routine treatments to routine treatments alone, a random effects model was used for two pooled trials $[47,59]$ because of statistical heterogeneity $\left(I^{2}=88 \%\right)$. The results showed that $\mathrm{CHM}$ combined with routine treatments was more effective than routine treatments alone (MD -8.14, $95 \%$ CI -15.70 to -0.58$)$. The subgroup analysis showed that MTQB combined with routine treatments (1 trial) [59] resulted in a statistically significant reduction in progesterone as compared to routine treatments alone. There were no significant differences observed in another trial [47] when MSHS was compared with a placebo (shown in Table 3 ).

3.3.5. Adverse Events. In this review, 8 trials [21, 28, 40, 45, $47,48,58,70]$ did not report any information regarding adverse events, and 39 trials [26, 29-39, 41-44, 46, 49-57, 59$69,71,72]$ mentioned adverse events. In trials comparing CHM with a placebo, one trial [57] reported no adverse events occurring in the intervention group. The placebo group, 
however, reported adverse events including dryness of mouth and constipation. One trial [63] reported the adverse event of mouth dryness in the $\mathrm{CHM}$ group. In trials comparing $\mathrm{CHM}$ with routine treatments alone, 5 trials $[42,50,62,64,65]$ reported adverse events occurring in both groups, and 4 trials $[34,35,71,72]$ reported no adverse events occurring in the CHM groups. Adverse events occurring in the CHM groups included gastric discomfort, nausea, dizziness, sleepiness, and constipation. In trials comparing CHM combined with routine treatments to routine treatments alone, 24 trials $[26,30-33,36-39,41,43,44,46,49,51,53-55,59-61,66$, $67,69]$ reported adverse events occurring in both groups. Two trials $[29,52]$ reported no adverse events occurring in CHM combined with routine treatments groups, and 2 trials $[56,68]$ reported no adverse events occurring in both groups. The adverse events occurring in the CHM combined with routine treatments group included loss of appetite, sleep disorders, nausea, vomiting, constipation, tachycardia, hypodynamia, dysphoria, tremor, dizziness, diarrhea, sleepiness, insomnia, headache, weight gain, hypotension, akathisia, blurred vision, chest distress, heart palpitations, skin rash, hyperhidrosis, abdominal discomfort, urination disorders, sexual dysfunction, transaminase elevation, blood system involvement, abnormal liver function, and abnormal ECG. 30 trials [26, 29-32, 34-36, 41-43, 46, 49-55, 57, 59, 60, 62-66, $69,71,72]$ reported a number of participants who experienced adverse events, 6 trials $[26,33,37,39,61,64]$ utilized TESS, and 2 trials $[38,44]$ utilized SERS to evaluate the adverse events. For better evaluation of the safety of TCM in the treatment of PPD, related data were analyzed.

According to a meta-analysis of the incidence of adverse events, 2 trials $[57,63]$ comparing CHM with a placebo warranted a fixed effects model for pooling analysis due to no statistical heterogeneity $\left(I^{2}=33 \%\right)$. The results revealed no significant differences between the two groups in reducing the incidence of adverse events (RR $0.67,95 \%$ CI 0.11 to 3.91). Subgroup analysis revealed no significant differences between MSHS (1 trial) [57] or MRLQS plus MTQB (1 trial) [63] and a placebo. For the 9 trials comparing CHM with routine treatments alone $[30,34,42,50,62,64,65,71,72]$ data was pooled and a random effects model was used because of statistical heterogeneity $\left(I^{2}=57 \%\right)$. The results showed that CHM had superior effectiveness to routine treatments alone in reducing the incidence of adverse events (RR 0.18, 95\% CI 0.09 to 0.38 ). Subgroup analysis showed that MTQB (2 trial) [34, 62] was more effective than routine treatments alone (RR 0.12, 95\% CI 0.04 to 0.36). MRLQS plus MSHS (3 trial) $[35,50,64,65]$ was more effective than routine treatments alone (RR $0.21,95 \%$ CI 0.10 to 0.47 ). MRLQS plus MTQB (1 trial) [71] or MRLQS plus MTQB plus MBASD (1 trial) [72] was more effective than routine treatments alone. No significant difference was observed when MRLQS plus MSHS plus MBASD (1 trial) [42] was compared with routine treatments alone. For the 19 trials [30-33, 36, 41, 43, 44, 46, $49,51-55,59,60,66,68,69]$ comparing CHM combined with routine treatments to routine treatments alone, a random effects model was used to pool data because of statistical heterogeneity $\left(I^{2}=56 \%\right)$. The results revealed that $\mathrm{CHM}$ combined with routine treatments had superior effectiveness to routine treatments alone in reducing the incidence of adverse events (RR $0.49,95 \%$ CI 0.37 to 0.65 ). Subgroup analysis of 9 trials [30, 31, 43, 44, 49, 51, 52, 55, 60] utilizing MRLQS combined with routine treatments showed that this treatment had superior effectiveness to routine treatments alone (RR $0.45,95 \%$ CI 0.32 to 0.64 ). Three trials [36, 41, 53] utilizing MRLQS plus MSHS combined with routine treatments showed that this treatment was more effective than routine treatments alone (RR $0.45,95 \%$ CI 0.22 to 0.93 ). Three trials $[46,59,66]$ involving MTQB combined with routine treatments, 2 trials $[32,69]$ involving MRLQS plus MTQB combined with routine treatments, and 2 trials [54, 68] involving MRLQS plus MBASD combined with routine treatments demonstrated no significant differences when compared to routine treatments alone (shown in Table 3).

Meanwhile, one trial [64] utilized TESS to compare MRLQS plus MSHS with routine treatments alone. The results showed that MRLQS plus MSHS was more effective than routine treatments alone. Five trials [33, 37, 39, 61, 69] compared CHM combined with routine treatments to routine treatments alone utilizing TESS. A random effects model was used to pool data because of statistical heterogeneity $\left(I^{2}=\right.$ $71 \%)$. The results showed that CHM combined with routine treatments had superior effectiveness to routine treatments alone in reducing TESS (MD $-1.80,95 \% \mathrm{CI}-2.46$ to -1.14 ). Subgroup analysis of two trials $[37,61]$ showed that MRLQS combined with routine treatments had superior effectiveness to routine treatments alone (MD $-2.31,95 \% \mathrm{CI}-3.12$ to $-1.50)$. MTQB combined with routine treatments (1 trial) [39], MRLQS plus MTQB combined with routine treatments (1 trial) [69], and MRLQS plus MBASD combined with routine treatments ( 1 trial) [33] were all shown to be more effective than routine treatments alone. A pooled analysis of 2 trials $[38,44]$ revealed that MRLQS combined with routine treatments had superior effectiveness to routine treatments alone in reducing SERS (MD $-5.19,95 \%$ CI -9.73 to -0.64 ) (shown in Table 3).

3.3.6. Publication Bias Assessment. A funnel plot for HAMD score following treatment with MRLQS combined with routine treatments in the experimental group and routine treatments alone in the control group was conducted to investigate the publication bias. The result indicated that publication bias may have existed in this review (Figure 3).

\section{Discussion}

4.1. Principal Findings. PPD has become a public health problem concern, as it is harmful to postpartum women and their families. Women have an important responsibility in the parenting of newborns. Therefore, selecting a safe and effective method of treatment is of particular importance. This study conducted a meta-analysis to assess the safety and efficacy of treatments for PPD using the therapeutic methods of TCM.

This review evaluated 47 trials and the pooled results demonstrated that CHM treatments alone or in combination 


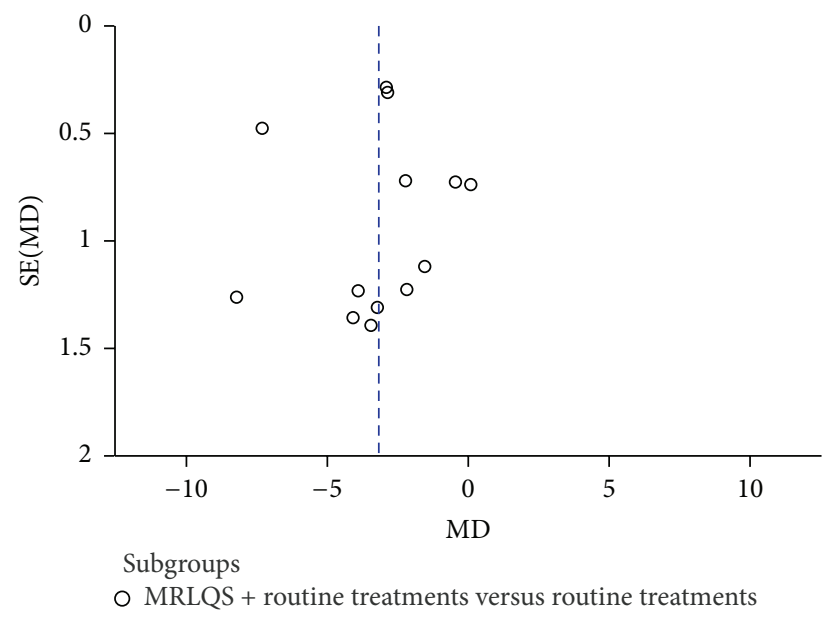

FIgURE 3: Funnel plot of comparison: MRLQS combined with routine treatments versus routine treatments alone; outcome: HAMD scores.

with routine treatments were effective in decreasing HAMD scores, EPDS scores, and incidence of adverse events. It indicated that CHM may be a safer and more effective treatment for PPD than routine treatments alone. A subgroup analysis was also conducted because the diversity of therapeutic methods of TCM used was an important factor influencing clinical effect. HAMD score was considered to be an important tool for evaluating the clinical efficacy of PPD. CHM plus routine treatments $(76.60 \%, 36 / 47)$ was the most common treatment approach. According to pooled data from related trials, the results suggest that MRLQS combined with routine treatments is more effective than MRLQS plus MSHS combined with routine treatments or MTQB combined with routine treatments in reducing HAMD score when each is compared with routine treatments alone. In this review, we also observed that MRLQS plus MSHS alone had a better clinical efficacy than routine treatments alone for PPD. However, it was difficult to evaluate other therapeutic methods of TCM individually or in combination with routine treatments because the data could not be pooled for analysis.

In the TCM theory, depression belongs to the category of emotion and will diseases. It is mainly caused by emotional triggers and viscera weakness. Stagnation of liver-qi caused by multiple etiologies is the most important mechanism of depression because of the role of the liver in regulating emotion. Meanwhile, postpartum women had the physiological characteristics of deficiency syndrome and stasis syndrome according to the TCM theory [68]. In the case of maternal frail state, excessive thinking exhausts the heart blood and spleen qi, which leads to deficiencies in both heart and spleen. Hypofunction of heart and spleen leads to mind (xin shen) dystrophy and causes or aggravates the symptoms of PPD. Stagnation of liver qi and deficiency of both heart and spleen are the principal pathogenic mechanisms of PPD. Therefore, stagnation of liver qi alone or in combination with deficiencies of both heart and spleen is the main type of syndrome differentiation (bian zheng fen xing) according to
TCM theory. MRLQS plus MSHS is a potential treatment option besides MRLQS alone for the management of PPD. This matches the principal pathogenesis and syndrome differentiation according to Chinese medicine. This study found that MRLQS plus MSHS in combination with routine treatments was clinically effective when compared with routine treatments alone in reducing HAMD score. Meanwhile, mean difference (MD) value of MRLQS plus MSHS in combination with routine treatments was greater than MRLQS plus MSHS alone, which suggests that a combination of MRLQS plus MSHS with routine treatments may have synergistic effects. Meta-analysis of EPDS further confirmed the effectiveness of MRLQS plus MSHS in combination with routine treatments. In terms of safety, MRLQS in combination with routine treatments or MRLQS plus MSHS in combination with routine treatments was associated with fewer adverse effects than routine treatments alone according to the existing data. Therefore, based on our analysis of clinical research and TCM theory, the use of MRLQS in combination with routine treatments or MRLQS plus MSHS in combination with routine treatments appears to be a superior approach for the treatment of PPD. Additionally, because MRLQS plus MSHS alone is safer and more effective than routine treatments, MRLQS plus MSHS might be an alternative option for patients who cannot tolerate antidepressant drugs.

In the present review, $47.22 \%$ of the trials (17/36) utilized MRLQS in combination with routine treatments for the management of PPD. Xiao yao powder (11/17) was identified as the most frequently used Chinese herbal compound in related studies. A prior systematic review revealed the effectiveness of Xiao yao powder in the treatment of depression [75]; further, it indicated that Xiao yao powder may be effective in the treatment of PPD. In trials evaluating MRLQS plus MSHS, shugan jieyu capsule was the most frequently used Chinese herbal compound, and a prior systematic review also reported evidence supporting the effectiveness of shugan jieyu capsule for the treatment of depression [76]. Although the present review confirmed the effectiveness of MRLQS or MRLQS plus MSHS and their corresponding Chinese herbal compounds, this information should be used cautiously. The therapeutic method of TCM and its corresponding Chinese herbal compound should be selected based on Chinese medicine syndrome differentiation (bian zheng). Due to the high risk of bias, the significant clinical heterogeneity observed between the trials, and the relatively small sample size, a definite conclusion still cannot be drawn.

4.2. Limitations of This Review. There were some limitations in this review which contributed to the inconclusive results. First, all trials were of poor methodological quality, and there was a high risk of bias. Of the 47 trials, only $42.55 \%$ (20/47) provided information on the methods for sequence generation, and most of them provided insufficient information to ascertain whether the randomization was conducted properly. The remainder of the trials only mentioned that "the patients were randomized into two groups." Allocation concealment was mentioned in only one trial [57]. Although the trial indicated that the random numbers had been placed 
in a sealed envelope, it was not mentioned whether the envelope was translucent. Only one trial [63] claimed doubleblind design and presample size estimation. The majority of the trials (72.34\%) did not provide any information regarding withdrawal from the study, and only one trial [57] conducted intention-to-treat analysis.

Second, the therapeutic methods of TCM and Chinese herbal compounds investigated may be responsible for the considerable heterogeneity in this review. In the practice of TCM, the herbalist should evaluate the patients symptoms to conduct syndrome differentiation and then propose the corresponding therapeutic methods of TCM. The herbal formulation is prescribed based on the therapeutic methods of TCM. This is called syndrome differentiation and treatment (bian zheng lun zhi), which is the strength of TCM. According to TCM theory, one type of syndrome differentiation should match one type of fixed therapeutic method of TCM. Different types of Chinese herbal formulations are prescribed to create individualized treatment plans for the patients under the guidance of the relatively fixed therapeutic methods of TCM. Although the specifics of CHM may differ among the trials, the therapeutic methods are consistent. Therefore, the therapeutic methods of TCM are at the core of CHM treatment. However, only $19.15 \%$ of the trials (9/47) provided information on the patients' type of syndrome differentiation, only $38.30 \%$ of the trials (18/47) provided information on the therapeutic methods of TCM, and only $12.77 \%$ of the trials (6/47) contained both. Meanwhile, 39 different Chinese herbs were investigated in this review and could be divided into 8 categories of therapeutic methods of TCM. The formulations included herbal formulas (27 trials), powder (2 trials), granules (2 trials), pills (6 trials), capsules (5 trials), herbal extracts ( 3 trials), and liquids (2 trials). Therefore, it was difficult to draw a definite conclusion regarding which therapeutic method of TCM or Chinese herbal compound was the most effective.

Third, the majority of the trials did not provide followup information. Three trials provided follow-up information over a 3-6-month period; however, this is considered insufficient for assessment of the long-term effectiveness of CHM.

Finally, this review found that reporting on adverse events in the included trials was inadequate. No mention of adverse events was made by $21.78 \%$ of the trials (10/47). Although $78.72 \%$ (37/47) of the trials reported adverse events, the information was limited. No trials reported whether CHM could affect breast-feeding or have adverse effects on newborns. Therefore, the safety of CHM for the treatment of PPD could not be assessed. Deficiencies in this systematic review limit the reliability of the meta-analysis results.

4.3. Implications of Further Research. To ensure evidencebased clinical practice, more rigorous multicenter, large, methodological design trials are warranted to provide higher quality evidence. The process of random sequence generation, adequate allocation concealment, double blinding, clear description of reasons for withdrawal, and intentionto-treat should be addressed. CHM should be conducted based on the syndrome differentiation pattern of PPD and the guidelines for TCM therapeutic methods, which have been previously reported. The adverse events associated with CHM should be described. Participants should be followed up for an extended period of time to assess the long-term effects of the treatment. Trials should adhere to CONSORT standards (http://www.consort-statement.org/) to promote internal and external validity.

\section{Conclusion}

In conclusion, the evidence indicates that $\mathrm{CHM}$ was effective in treating PPD; additionally, MRLQS combined with routine treatments or MRLQS plus MSHS combined with routine treatments was more effective than routine treatments alone. MRLQS plus MSHS alone might be an effective alternative to routine treatments. However, the evidence detailed in this review should be considered cautiously because of the high risk of bias and the poor methodological quality of the trials. Therefore, rigorously designed trials are needed for further evaluation of the safety and efficacy of CHM for the treatment of PPD.

\section{Competing Interests}

All authors declare that they have no conflict of interests.

\section{Authors' Contributions}

Yongle Li designed the review, analyzed data, and drafted the manuscript. Zijie Chen and Ning Yu searched, selected articles, and made substantial revisions to the paper. Keyu Yao and Yupeng Xi extracted data and assessed the quality of the methodology. Yiwen Che analyzed data and drafted the manuscript. Shuangqing Zhai designed and made substantial revisions to the paper.

\section{Acknowledgments}

This review is funded by the Independent Research Project of BUCM and the Specialized Research Fund for the Doctoral Program of Higher Education (20120013110002).

\section{References}

[1] P. Almond, "Postnatal depression: a global public health perspective," Perspectives in Public Health, vol. 129, no. 5, pp. 221227, 2009.

[2] J. F. Paulson and S. D. Bazemore, "Prenatal and postpartum depression in fathers and its association with maternal depression: a meta-analysis," The Journal of the American Medical Association, vol. 303, no. 19, pp. 1961-1969, 2010.

[3] S. L. Grace, A. Evindar, and D. E. Stewart, "The effect of postpartum depression on child cognitive development and behavior: a review and critical analysis of the literature," Archives of Women's Mental Health, vol. 6, no. 4, pp. 263-274, 2003.

[4] D. F. Hay, S. Pawlby, D. Sharp, P. Asten, A. Mills, and R. Kumar, "Intellectual problems shown by 11-year-old children whose mothers had postnatal depression," The Journal of Child Psychology and Psychiatry, vol. 42, no. 7, pp. 871-889, 2001. 
[5] L. Murray, D. Sinclair, P. Cooper, P. Ducournau, P. Turner, and A. Stein, "The socioemotional development of 5-year-old children of postnatally depressed mothers," Journal of Child Psychology and Psychiatry and Allied Disciplines, vol. 40, no. 8, pp. 1259-1271, 1999.

[6] U. Halbreich and S. Karkun, "Cross-cultural and social diversity of prevalence of postpartum depression and depressive symptoms," Journal of Affective Disorders, vol. 91, no. 2-3, pp. 97-111, 2006.

[7] D. D. Affonso, A. K. De, J. A. Horowitz, and L. J. Mayberry, "An international study exploring levels of postpartum depressive symptomatology," Journal of Psychosomatic Research, vol. 49, no. 3, pp. 207-216, 2000.

[8] C. T. Beck, "Predictors of postpartum depression: an update," Journal of Nursing Research, vol. 50, no. 5, pp. 275-285, 2001.

[9] A.-W. Deng, R.-B. Xiong, T.-T. Jiang, Y.-P. Luo, and W.-Z. Chen, "Prevalence and risk factors of postpartum depression in a population-based sample of women in Tangxia Community, Guangzhou," Asian Pacific Journal of Tropical Medicine, vol. 7, no. 3, pp. 244-249, 2014.

[10] J. Eastwood, L. Kemp, and B. Jalaludin, "Explaining ecological clusters of maternal depression in South Western Sydney," BMC Pregnancy and Childbirth, vol. 14, article 47, 15 pages, 2014.

[11] I. S. Yim, L. M. Glynn, C. D. Schetter, C. J. Hobel, A. ChiczDeMet, and C. A. Sandman, "Risk of postpartum depressive symptoms with elevated corticotropin- releasing hormone in human pregnancy," Archives of General Psychiatry, vol. 66, no. 2, pp. 162-169, 2009.

[12] A. Taylor, V. Glover, M. Marks, and M. Kammerer, "Diurnal pattern of cortisol output in postnatal depression," Psychoneuroendocrinology, vol. 34, no. 8, pp. 1184-1188, 2009.

[13] E. Comasco, S. M. Sylvén, F. C. Papadopoulos, I. SundströmPoromaa, L. Oreland, and A. Skalkidou, "Postpartum depression symptoms: a case-control study on monoaminergic functional polymorphisms and environmental stressors," Psychiatric Genetics, vol. 21, no. 1, pp. 19-28, 2011.

[14] M. Bloch, P. J. Schmidt, M. Danaceau, J. Murphy, L. Nieman, and D. R. Rubinow, "Effects of gonadal steroids in women with a history of postpartum depression," The American Journal of Psychiatry, vol. 157, no. 6, pp. 924-930, 2000.

[15] T. C. Couto, M. Y. Brancaglion, A. Alvim-Soares et al., "Postpartum depression: a systematic review of the genetics involved," World Journal of Psychiatry, vol. 5, no. 1, pp. 103-111, 2015.

[16] C.-L. Dennis, "Psychosocial interventions for the treatment of perinatal depression," Best Practice \& Research: Clinical Obstetrics \& Gynaecology, vol. 28, no. 1, pp. 97-111, 2014.

[17] F. De Crescenzo, F. Perelli, M. Armando, and S. Vicari, "Selective serotonin reuptake inhibitors (SSRIs) for post-partum depression (PPD): a systematic review of randomized clinical trials," Journal of Affective Disorders, vol. 152-154, no. 1, pp. 39-44, 2014.

[18] R. C. Ng, C. K. Hirata, W. Yeung, E. Haller, and P. R. Finley, "Pharmacologic treatment for postpartum depression: a systematic review," Pharmacotherapy, vol. 30, no. 9, pp. 928-941, 2010.

[19] C.-L. Dennis, L. E. Ross, and A. Herxheimer, "Oestrogens and progestins for preventing and treating postpartum depression," Cochrane Database of Systematic Reviews, no. 4, Article ID CD001690, 2008.

[20] M. Huang, "Study of gui pi decotion combined with psychological intervention treating 50 cases of postpartum depression," Hunan Journal of Traditional Chinese Medicine, vol. 29, no. 7, pp. 64-65, 2013 (Chinese).
[21] X. Ran, "Clinical efficacy study of dan zhi xiao yao powders with small dose of venlafaxine for the treatment of postpartum depression," Chinese Journal of Clinical Rational Drug Use, vol. 6, no. 12, p. 73, 2013 (Chinese).

[22] N. Zhang and J. H. Wu, "Treatment of postpartum depression from elective prescription based on the therapeutical methods of liver," Journal of Hubei University of Chinese Medicine, vol. 15, no. 6, pp. 40-41, 2013 (Chinese).

[23] Y. P. Li, "Curative effect observation of da ding feng zhu treating 38 cases of postpartum depression," Study Journal of Traditional Chinese Medicine, vol. 23, no. 8, p. 1491, 2005 (Chinese).

[24] L. X. Hong, L. Chen, and Y. Zhang, "A comparative study of ban xia hou pu decoction combined with citalopram in treatment of patients with postpartum depression," Journal of Psychiatry, vol. 25, no. 1, pp. 45-47, 2012 (Chinese).

[25] X. Q. Zheng, "Clinical observation on the treatment of 52 cases of postpartum depression with traditional Chinese and western medicine," Guiding Journal of Guiding Journal of Traditional Chinese Medicine and Pharmacy, vol. 13, no. 6, pp. 50-51, 2007 (Chinese).

[26] P. Zheng, "Clinical efficacy and safety evaluation of integrated traditional Chinese and western medicine for treating postpartum depression," Journal of New Chinese Medicine, vol. 47, no. 12, pp. 135-136, 2015 (Chinese).

[27] S. S. Yang, R. Fan, and J. H. Guo, "Meta-analysis on clinical effects of integrated traditional Chinese and western medicine for treating postpartum depression," Chinese General Practice, vol. 27, no. 18, pp. 2763-2765, 2011 (Chinese).

[28] X. J. Cai, "Clinical comparative study of Chinese medicine intervention for postpartum depression," Hebei Medical Journal, vol. 37, no. 12, pp. 1830-1833, 2015 (Chinese).

[29] Z. B. Chen, Z. J. Ma, and F. H. Nie, "Clinical observation of $\mathrm{fu}$ lin shen zhi shuang xin pill treating 34 cases of postpartum depression," Hebei Journal of Traditional Chinese Medicine, vol. 37, no. 6, pp. 844-845, 2015 (Chinese).

[30] G. L. Chen, X. X. Song, and X. P. Zhang, "Study of selfmade qing yu ping xin decoction in treating 38 cases of postpartum depression," International Journal of Traditional Chinese Medicine, vol. 33, no. 8, pp. 733-734, 2011 (Chinese).

[31] G. A. Ding, G. H. Yu, S. C. Liang et al., "Clinical effect of jia wei xiao yao powder combined with mirtazapine in the treatment of postpartum depression," Guangdong Medical Journal, vol. 31, no. 16, pp. 2163-2165, 2010 (Chinese).

[32] H. Y. Fan, "Study on postpartum depression treated with combination of TCM and western medicine," Medical Information, vol. 24, no. 2, pp. 772-773, 2011 (Chinese).

[33] B. H. Fang, "Clinical curative effect observation of tiao xue jie yu decoction and venlafaxine in treatment of postpartum depression," Chinese Journal of Traditional Medical Science and Technology, vol. 21, no. 6, pp. 666-667, 2014 (Chinese).

[34] H. S. Gao, "Clinical curative effect observation of yu lin zhu decoction modification in the treatment of postpartum depression," Hubei Journal of Traditional Chinese Medicine, vol. 32, no. 4, pp. 42-43, 2010 (Chinese).

[35] J. H. Guo, S. S. Wang, and R. Fan, "Curative effect observation of chai hu shu gan san and gan mai da zao decoction in the treatment of postpartum depression," Journal of North Pharmacy, vol. 8, no. 2, pp. 18-20, 2011 (Chinese).

[36] R. J. Hao, "Controlled study of shu gan jie yu capsule combined with citalopram in the treatment of postpartum depression," Chinese Remedies \& Clinics, vol. 15, no. 9, pp. 1315-1317, 2015 (Chinese). 
[37] H. He and L. R. Ren, "Clinical curative effect observation of xiao yao san modification combined with fluoxetine in the treatment of postpartum depression," Hubei Journal of Traditional Chinese Medicine, vol. 30, no. 12, pp. 37-38, 2008 (Chinese).

[38] F. Y. Hu, "Clinical efficacy and adverse events of xiao yao pill combined with fluoxetine in the treatment of postpartum depression," Health World, vol. 2013, no. 32, pp. 275-276, 2013 (Chinese).

[39] Y. H. Jiang, Q. H. Ye, Z. B. Chen et al., "Zhen yuan capsule and duloxetine in the treatment of postnatal depression," China Journal of Health Psychology, vol. 20, no. 10, pp. 1494-1495, 2012 (Chinese).

[40] X. D. Jin and Z. H. Ying, "Clinical efficacy observation of shu gan jie yu capsule and paroxetine in the treatment of postpartum depression," Chinese Remedies \& Clinics, vol. 13, no. 6, pp. 798-799, 2013 (Chinese).

[41] P. P. Lei and L. W. Fu, "Clinical curative effect observation of integrated traditional Chinese and western medicine for treating postpartum depression," Modern Journal of Integrated Traditional Chinese and Western Medicine, vol. 24, no. 23, pp. 2582-2583, 2015 (Chinese).

[42] S. H. Li, J. Q. Li, W. Q. Li et al., "Nursing countermeasures and the evaluation of the curative effect used with traditional Chinese medicine on postpartum depression," Chinese Journal of Ethnomedicine and Ethnopharmacy, vol. 22, no. 14, pp. 161163, 2013 (Chinese).

[43] G. D. Li and W. Zhang, "Clinical observation of integrated traditional Chinese and western medicine for treating postpartum depression," Chinese Journal of Healthy Birth \& Child Care, vol. 20, no. 5, pp. 321-323, 2014 (Chinese).

[44] Q. Y. Liang, Y. H. Xie, X. R. Wu et al., "Evaluation on clinical efficacy and safety of xiao yao pills combined with fluoxetine in treating postpartum depression," Journal of Modern Medicine \& Health, vol. 28, no. 13, pp. 1972-1975, 2012 (Chinese).

[45] H. Lin, X. Ye, Z. P. Guo et al., "Combination of paroxetine and xiao yao san for postpartum depression," Applied Journal of General Practice, vol. 6, no. 5, pp. 476-477, 2008 (Chinese).

[46] G. L. Liu, "Huo li su oral liquid combined with sertraline in the treatment of patients with postpartum depression due to qiblood deficiency," International Journal of Traditional Chinese Medicine, vol. 37, no. 3, pp. 228-231, 2015 (Chinese).

[47] Y. Lv, Clinical Study of the Therapy of Invigorating Heart and Spleen to Treat Postpartum Depression, Guangzhou University of Chinese Medicine, Guangzhou, China, 2007 (Chinese).

[48] H. R. Mi, W. R. Zhang, Q. Zhang et al., "Clinical observation on strengthening spleen and regulating liver for the treatment of 86 cases of postpartum depression," Hebei Journal of Traditional Chinese Medicine, vol. 36, no. 9, pp. 1310-1311, 2014 (Chinese).

[49] H. P. Pan and G. X. Wu, "A control study of dan zhi xiao yao san combined with small dose venlafaxine in the treatment of postpartum depression," Journal of Clinical Psychosomatic Diseases, vol. 19, no. 2, pp. 119-123, 2013 (Chinese).

[50] S. F. Qian, "Clinical observation of shu gan jie yu capsule for the treatment of postpartum depression," Journal of New Chinese Medicine, vol. 46, no. 12, pp. 84-85, 2014 (Chinese).

[51] X. M. Ren, "Clinical curative effect observation of integrated traditional Chinese and western medicine for treating 30 cases of postpartum depression," China Medical Herald, vol. 6, no. 10, p. 103, 2009 (Chinese).

[52] A. Q. Shao, M. X. Guo, and X. M. Xu, "Clinical effect of xiao chai hu decoction assisting paroxetine in the treatment of postpartum depression," Chinese Primary Health Care, vol. 25, no. 9, pp. 81-82, 2011 (Chinese).

[53] Y. L. Shi, "Clinical observation on the treatment of postpartum depression with Chinese and western medicine," Henan Journal of Tradition Chinese Medicine, vol. 33, no. B4, p. 152, 2013 (Chinese).

[54] X. Su, Clinical Study of Postnatal Depression Treated by Shu Gan Jie Yu Decoction Combined with Paroxetine, Hebei Medical University, Shijiazhuang, China, 2014 (Chinese).

[55] L. P. Sun, "Clinical study on fluoxetine combined with xiaoyao powder for the treatment of 32 cases of postpartum depression," Medical Innovation of China, vol. 9, no. 12, pp. 41-42, 2012 (Chinese).

[56] C. X. Wang, G. L. Chen, and M. Long, "Study on Jie yu decoction in treating 27 cases of postpartum depression," Chinese Journal of Experimental Traditional Medical Formulae, vol. 17, no. 9, p. 292, 2011 (Chinese).

[57] D. Wang, Clinical Efficacy Evaluation of Method of Strengthening Heart and Spleen Treating Postpartum Depression, Beijing University of Chinese Medicine, Beijing, China, 2012 (Chinese).

[58] X. M. Wang, B. Chen, and D. Wang, "Clinical observation of jie yu rou gan decoction for the treatment of postpartum depression," Guiding Journal of Traditional Chinese Medicine and Pharmacy, vol. 21, no. 13, pp. 74-76, 2015 (Chinese).

[59] Y. Q. Wang, L. Chen, Q. X. Teng et al., "A research of wu ling capsule combined with sertraline in the treatment of postpartum depression and the change of hormone and neurotransmitter in vivo," Journal of Zhejiang Chinese Medical University, vol. 38, no. 3, pp. 250-254, 2014 (Chinese).

[60] C. L. Wei, Y. Y. Ma, G. Z. Hong et al., "A clinical contrast study of sertraline and xiao yao pill on postpartum depression," Medical Journal of Chinese People's Health, vol. 21, no. 11, pp. 131-1318, 2009 (Chinese).

[61] L. Wu, Q. Wu, J. Li et al., "Sertraline joint at large clinical observation on treatment of postpartum depression," Jilin Journal of Traditional Chinese Medicine, vol. 34, no. 5, pp. 469-471, 2014 (Chinese).

[62] C. F. Xu, L. H. Yuan, and Q. Y. Wang, "Curative effect observation of bu xin pill treating 30 cases of postpartum depression," Shandong Journal of Traditional Chinese Medicine, vol. 25, no. 1, pp. 28-29, 2006 (Chinese).

[63] F. Xu, Q. S. Tang, and X. L. Li, "Replenishing kidney and regulating qi therapy for postpartum depression: a randomized controlled trial," Beijing Journal of Traditional Chinese Medicine, vol. 32, no. 3, pp. 200-203, 2013 (Chinese).

[64] B. J. Zhang, Y. Y. Ma, H. Y. Wang et al., "Clinical curative effect observation of Shu gan jie yu capsule and fluoxetine in treatment of postpartum depression," China Modern Medicine, vol. 21, no. 23, pp. 134-135, 2014 (Chinese).

[65] X. Q. Zhang, "Comparisons of clinical curative effect of citalopram and shu gan jie yu capsule in the treatment of postpartum depression," Medical Information, vol. 28, no. 7, pp. 331-332, 2015 (Chinese).

[66] X. M. Zhang and C. L. Liu, "Clinical observation on wu ling capsule combined fluoxetine in treating postpartum depression," Journal of New Chinese Medicine, vol. 41, no. 12, pp. 28-29, 2009 (Chinese).

[67] X. P. Zhao and H. Lin, "Study on fluoxetine combined with chai hu shu gan powder for the treatment of postpartum depression," Liaoning Journal of Traditional Chinese Medicine, vol. 33, no. 5, pp. 586-587, 2006 (Chinese). 
[68] L. C. Zheng, Randomized, Parallel Controlled Clinical Observational Study on Shen-Gui-Ren Decoction in Treating the Postpartum Depression of the Syndrome of Qi Xu Xue Xu Xue $Y u$ Complex, Chendu University of Chinese Medicine, Chendu, China, 2013 (Chinese).

[69] M. Y. Zhou, "Clinical study of yang xue ning shen decoction combined with sertraline in the treatment of postpartum depression," Journal of Medical Forum, vol. 34, no. 11, pp. 126127, 2013 (Chinese).

[70] H. X. Zhou, W. Z. Liao, M. Y. Wang et al., "Yue ju pills and gan mai da zao treating postpartum depression," Medical Innovation of China, vol. 12, no. 6, pp. 104-105, 2015 (Chinese).

[71] L. P. Zhu, "Observation on effect of yang xin jie yu decoction in the treatment of postpartum depression," Chinese Primary Health Care, vol. 22, no. 2, pp. 86-87, 2008 (Chinese).

[72] J. P. Zhu, "Clinical curative effect observation of suan zao ren decoction modification in treatment of postpartum depression," Journal of New Chinese Medicine, vol. 46, no. 7, pp. 105-106, 2014 (Chinese).

[73] J. J. Deeks, J. P. Higgins, and D. G. Altman, "Assessing risk of bias in included studies," in Cochrane Handbook for Systematic Reviews of Interventions Version 5.1.0, J. Higgins and S. Green, Eds., chapter 8, The Cochrane Collaboration, 2011, http://www.cochrane-handbook.org/.

[74] J. J. Deeks, J. P. Higgins, and D. G. Altman, "Analyzing data and undertaking meta-analyses," in Cochrane Handbook for Systematic Reviews of Interventions Version 5.1.0, J. Higgins and S. Green, Eds., chapter 9, The Cochrane Collaboration, 2011, http://www.cochrane-handbook.org/.

[75] X. K. Qin, P. Li, M. Han et al., "Systematic review of randomize controlled trials of Xiao yao powder in treatment of depression," Journal of Traditional Chinese Medicine, vol. 51, no. 6, pp. 500505, 2010 (Chinese).

[76] J. Cai, A Systematical Review of Shugan Jieyu Capsule (An' Jiaxin Capsule) for Depression, Chendu University of Chinese Medicine, Chendu, China, 2013 (Chinese). 


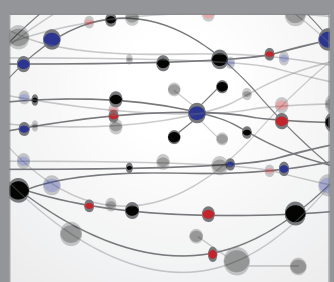

The Scientific World Journal
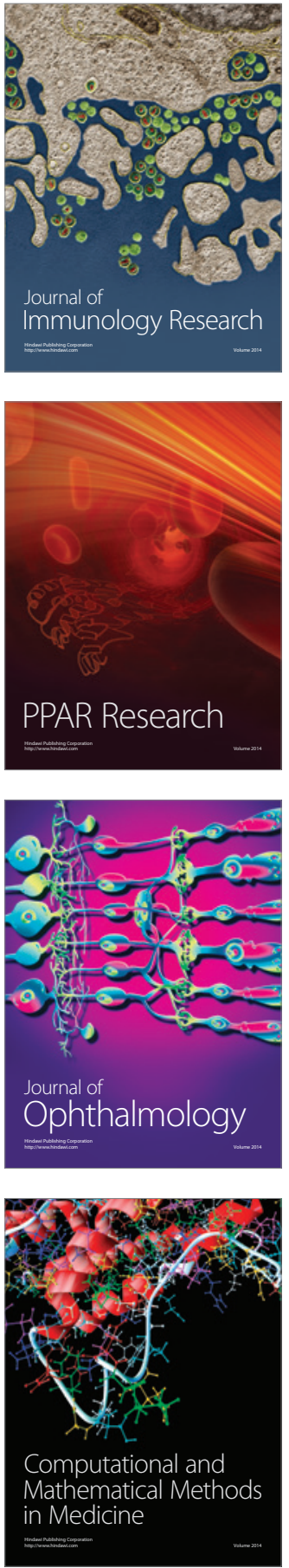

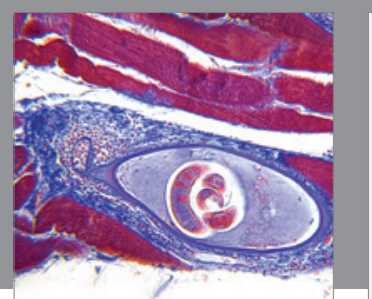

Gastroenterology Research and Practice

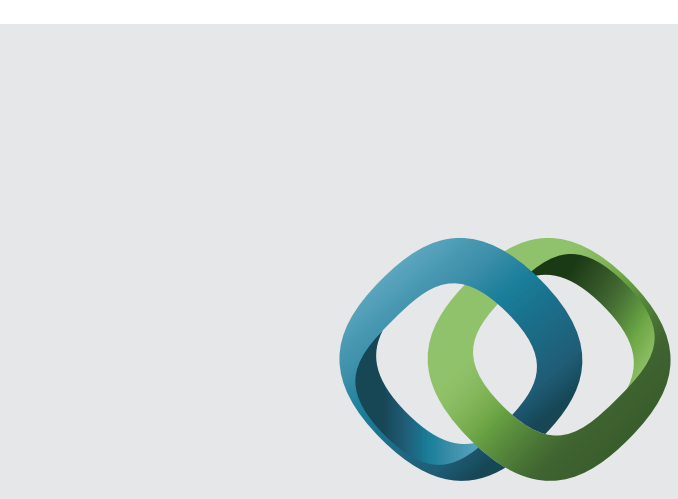

\section{Hindawi}

Submit your manuscripts at

http://www.hindawi.com
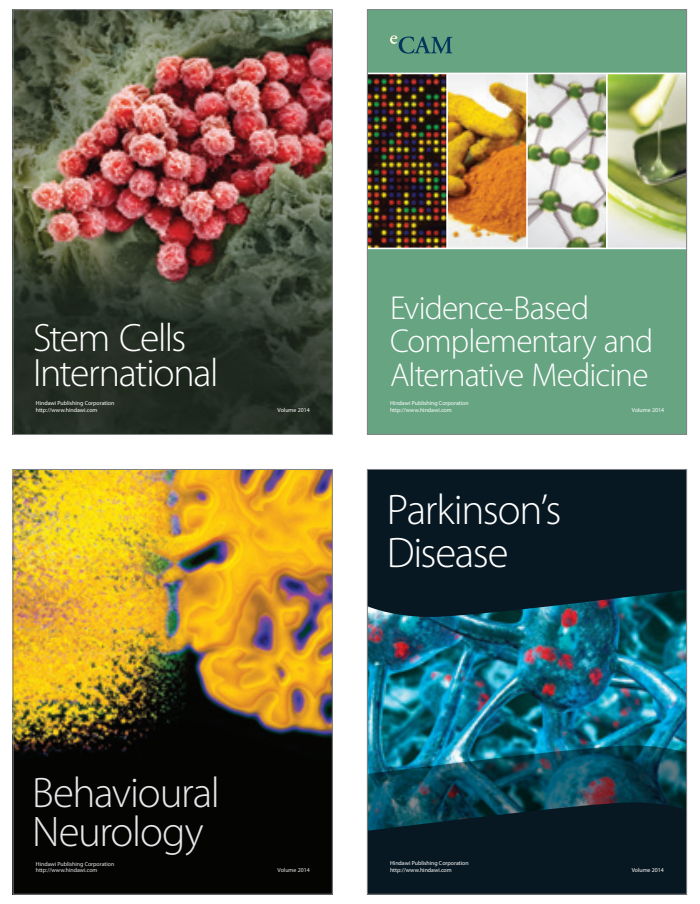
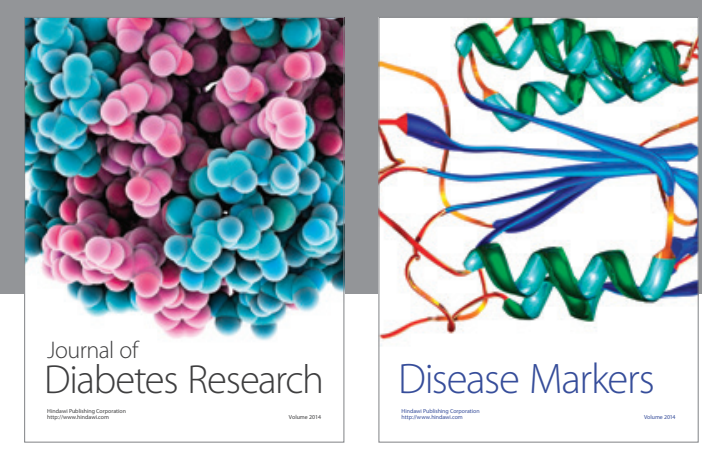

Disease Markers
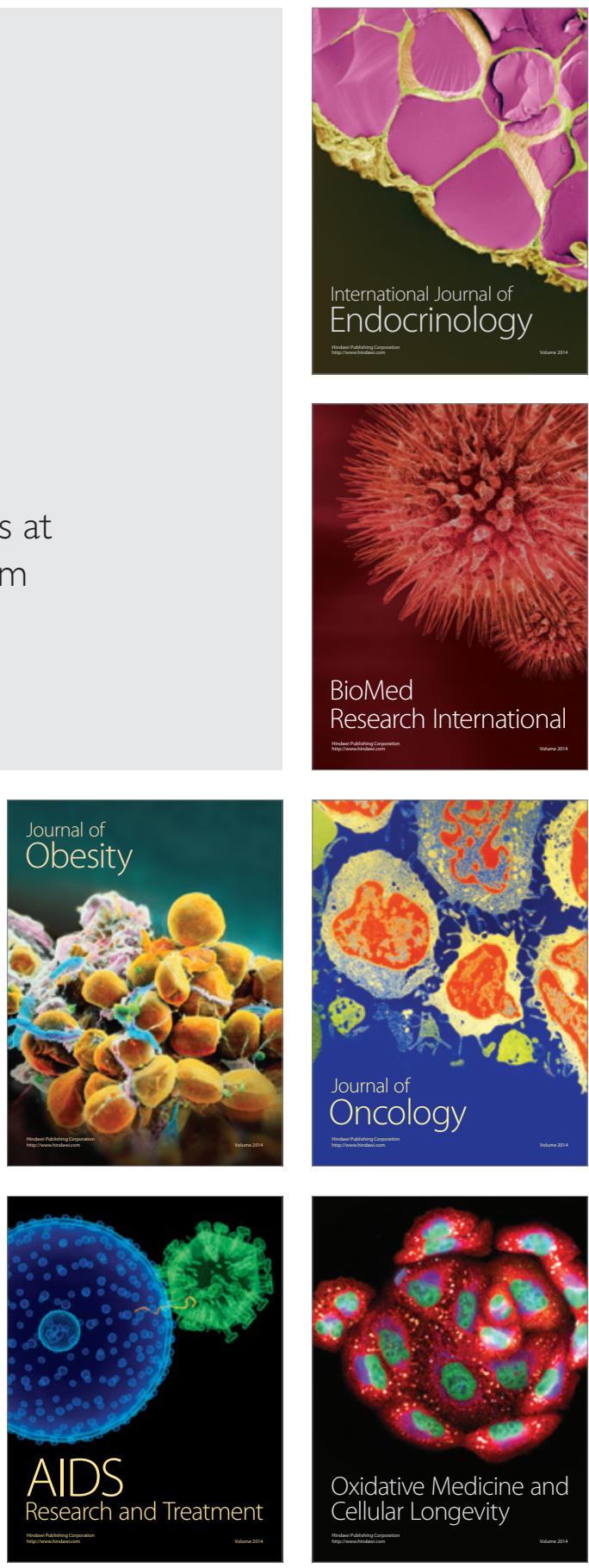